\title{
A FUZZY EPQ MODEL FOR NON-INSTANTANEOUS DETERIORATING ITEMS WHERE PRODUCTION DEPENDS ON DEMAND WHICH IS PROPORTIONAL TO POPULATION, SELLING PRICE AS WELL AS ADVERTISEMENT
}

\author{
Abhishek Kanti Biswas \\ R. K. M. Vivekananda Centenary College, India \\ E-mail: a.k.biswas@hotmail.com \\ Sahidul Islam \\ University of Kalyani, India \\ E-mail: sahidul.math@gmail.com
}

Submission: $11 / 03 / 2018$

Revision: $12 / 06 / 2018$

Accept: 12/20/2018

\section{ABSTRACT}

The inventory system has been drawing more intrigue because this system deals with the decision that minimizes the total average cost or maximizes the total average profit. For any farm, the demand for any items depends upon population, selling price and frequency of advertisement etc. Most of the model, it is assumed that deterioration of any item in inventory starts from the beginning of their production. But in reality, many goods are maintaining their good quality or original condition for some time. So, price discount is availed for defective items. Our target is to calculate the total optimal cost and the optimal inventory level for this inventory model in a crisp and fuzzy environment. Here Holding cost taken as constant and no-shortages are allowed. The cost parameters are considered as Triangular Fuzzy Numbers and to defuzzify the model Signed Distance Method is applied. A numerical example of the optimal solution is given to clarify the model. The changes of different parameters effect on the optimal total cost are presented and sensitivity analysis is given.

Keywords: EPQ Inventory, Non-Instantaneous Deterioration, Demand dependent Production, Defuzzification, Signed Distance Method 
INDEPENDENT JOURNAL OF MANAGEMENT \& PRODUCTION (IJM\&P)

http://www.ijmp.jor.br

V. 10, n. 5, September-October 2019

ISSN: 2236-269X

DOI: 10.14807/ijmp.v10i5.897

\section{INTRODUCTION}

In an EPQ inventory, it is important to control quality. Most of the models of the inventory control system are formulated with the assumption that all produced items are of good quality. But in reality, for any production company to produce all good quality products is impossible. On the other hand, due to the different phenomenon, there are so many goods which deteriorate after their lifetime. In such situation price discount are common practices by the supplier that encourages the customer to purchase defective and deteriorated items other than regular purchase. So the effect of deterioration and defective items cannot be ignored in inventory models.

Most inventory models considered the request rate to be either stock needy or consistent or time-subordinate. It has been observed that decrease in the cost of the item for the most part positively affects request of the item. It becomes a necessity to make a proper strategy to maintain the inventory economically.

Ghare et al. (1963) developed an inventory model for the exponentially decaying inventory system. These types of models were extended and improved by Misra (1975). The investigators generally have taken the demand as constant. In reality, demand always depends on selling price of an item, population of that area, deterioration, the frequency of advertisement of the product etc. As time advanced, a few researchers created inventory models with deteriorating items, shortage items, demand patterns, cost patterns, items order cycles and their combinations.

Bhunia et al. (2014) derived a deterministic inventory model where deteriorated items demand depends upon selling price of items and the frequency of advertisement. Ghoreishi et al. (2014) researched on an inventory model for noninstantaneous deteriorating items with partial backlogging, permissible delay in payments, inflation- and selling price-dependent demand and customer returns. On the other hand, to reduce the cost, an intelligent businessman or a production company always produce products depends on demand.

Without any ambiguity, many inventory model based on different kinds of vulnerabilities are classically modelled using the approaches from the probability hypothesis. Some of the business fit such conditions, yet applying these models as they may be, for the most part, prompts incorrect choices. Here fuzzy inventory models 
INDEPENDENT JOURNAL OF MANAGEMENT \& PRODUCTION (IJM\&P)

http://www.ijmp.jor.br

V. 10, n. 5, September-October 2019

ISSN: 2236-269X

DOI: 10.14807/ijmp.v10i5.897

fulfil that gap. We can get more exact outcomes for inventory problems, rather than the conventional likelihood hypothesis by using fuzzy set theory.

It was presented by Zadeh (1965) whose research work has been receiving considerable attention from investigators in production and inventory system. Bellmann et al. (1970) proposed a scientific model on decision making in fuzzy condition. Later, Dubois et al. (1978) defined some operations on fuzzy numbers. Zimmermann (1985) made an attempt to use the fuzzy sets in operation research. Syed et al. (2007) investigated a fuzzy inventory model without shortages using signed distance method.

Dutta et al. (2012) contributed on fuzzy inventory model without shortage using trapezoidal fuzzy number. Maragatham et al. (2014) researched on a fuzzy inventory model for deteriorating items with price-dependent demand. Islam and Biswas (2017) studied on a fuzzy inventory model having exponential demand with weibull distribution for non-instantaneous deterioration, shortages under partially backlogging and time dependent holding cost.

\subsection{Motivation \& Contribution of Study}

In the proposed model, we have shown a fuzzy deterministic stock model for non-instantaneous deteriorating things with production proportional to demand and variable demand pattern depends on population, selling price and frequency of advertisement which is variables or constants according to any real-life situation. Here we treated those as constants.

So, any production company produces any items according to demand. On the other hand, defection and deterioration occur for any production. In such situation price discount is a common phenomenon. The inventory parameters are taken as the triangular fuzzy number. Signed distance method is used to defuzzify the model. The goal for finding the solution for minimizing the total cost has been derived. To the author's best of knowledge such type of model has not yet been discussed in the inventory literature.

\section{DEFINITIONS AND FUZZY PRELIMINARIES}

Definition 2.1: A fuzzy set $\widetilde{A}$ is a universe of discourse $X$ is defined as the following set of pairs $\widetilde{A}=\left\{\left(x, \mu_{\widetilde{A}}(x): x \in X\right\}\right.$. Where $\mu_{\widetilde{A}}(x) \rightarrow[0,1]$ is a mapping called 
INDEPENDENT JOURNAL OF MANAGEMENT \& PRODUCTION (IJM\&P)

http://www.ijmp.jor.br

V. 10, n. 5, September-October 2019

ISSN: 2236-269X

DOI: 10.14807/ijmp.v10i5.897

the membership function of the set $\widetilde{\mathrm{A}}$ and $\mu_{\widetilde{A}}(\mathrm{x})$ is called the membership value or degree of membership of $x \in X$ in the fuzzy set $\widetilde{A}$. The larger $\mu_{\widetilde{A}}(x)$ is stronger the grade of membership form in $\widetilde{A}$.

Definition 2.2: A fuzzy set $\widetilde{A}$ of the universe of discourse $X$ is convex if and only if for all $x_{1}, x_{2} \in X, \mu_{\widetilde{A}}\left(\lambda x_{1}+(1-\lambda) x_{2}\right) \geq \min \left[\mu_{\widetilde{A}}\left(x_{1}\right), \mu_{\widetilde{A}}\left(x_{2}\right)\right]$ when $0 \leq \lambda \leq$ 1.

Definition 2.3: A fuzzy set $\widetilde{A}$ of the universe of discourse $X$ is called normal fuzzy set implying that there exists at least one $\mathrm{x} \in \mathrm{X}$ such that $\mu_{\widetilde{\mathrm{A}}}(\mathrm{x})=1$.

Definition 2.4: The $\alpha$ - cut of $\widetilde{A}$ is defined as a crisp set $A_{\alpha}=\left\{x: \mu_{\widetilde{A}}(x) \geq \alpha, x \in\right.$ $X$ where $\alpha \in[0,1] . A_{\alpha}$ is a non-empty bounded closed interval contained in $X$ and it can be denoted by $A_{\alpha}=\left[A_{L}(\alpha), A_{R}(\alpha)\right]$. Where $A_{L}(\alpha)$ and $A_{R}(\alpha)$ are the lower and upper bounds of the closed interval respectively.

Definition 2.5: A fuzzy number is a fuzzy set in the universe of discourse $X$ that is both convex and normal. The following figure (3) shows a fuzzy number $\widetilde{A}$.

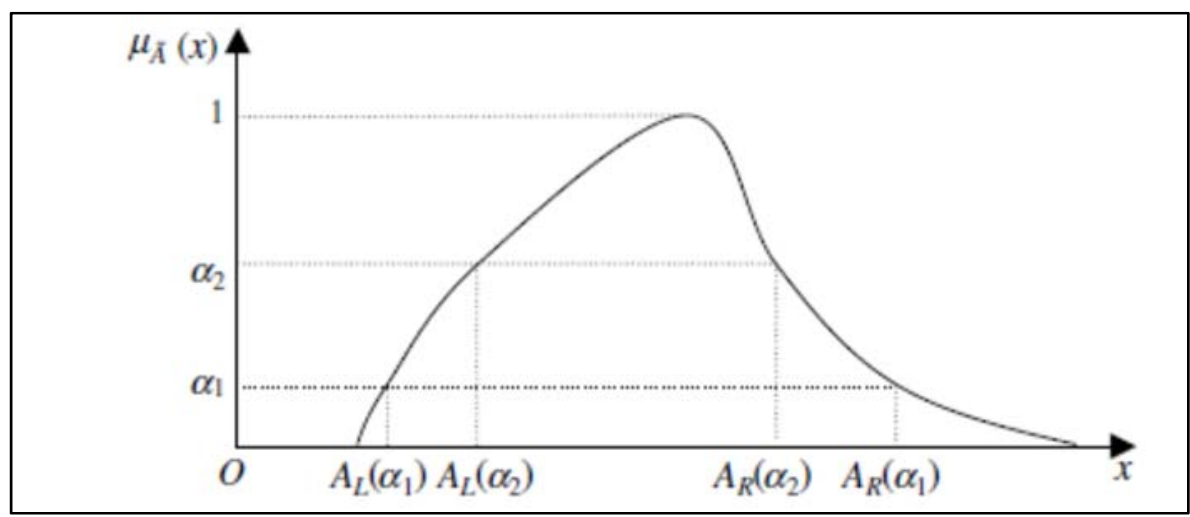

Figure 1: Fuzzy number $\widetilde{\mathrm{A}}$ With $\alpha$-cuts.

Above figure-1, shows a fuzzy number $\widetilde{A}$ with $\alpha$-cuts $A_{\alpha_{1}}=\left[A_{L}\left(\alpha_{1}\right), A_{R}\left(\alpha_{1}\right)\right]$, $A_{\alpha_{2}}=\left[A_{L}\left(\alpha_{2}\right), A_{R}\left(\alpha_{2}\right)\right]$. It is seen that if $\alpha_{2} \geq \alpha_{1}$ then $A_{L}\left(\alpha_{2}\right) \geq A_{L}\left(\alpha_{1}\right)$ and $A_{R}\left(\alpha_{2}\right) \geq$ $A_{R}\left(\alpha_{1}\right)$.

Definition 2.6: The function principle is used for the operation for Addition, Subtraction, Multiplication and Division of fuzzy numbers. Suppose $\widetilde{A}=\left(a_{1}, a_{2}, a_{3}\right)$ and $\widetilde{\mathrm{B}}=\left(\mathrm{b}_{1}, \mathrm{~b}_{2}, \mathrm{~b}_{3}\right)$ are two triangular fuzzy numbers. Then_ 
(i) Addition: $\widetilde{\mathrm{A}}+\widetilde{\mathrm{B}}=\left(\mathrm{a}_{1}+\mathrm{b}_{1}, \mathrm{a}_{2}+\mathrm{b}_{2}, \mathrm{a}_{3}+\mathrm{b}_{3}\right)$, where $\mathrm{a}_{1}, \mathrm{a}_{2}, \mathrm{a}_{3} ; \mathrm{b}_{1}, \mathrm{~b}_{2}$, $b_{3}$ are any real numbers.

(ii) Subtraction: $\widetilde{A}-\widetilde{B}=\left(a_{1}-b_{1}, a_{2}-b_{2}, a_{3}-b_{3}\right)$, where $a_{1}, a_{2}, a_{3} ; b_{1}, b_{2}$, $b_{3}$ are any real numbers.

(iii) Multiplication: $\widetilde{\mathrm{A}} \times \widetilde{\mathrm{B}}=\left(\mathrm{a}_{1} \mathrm{~b}_{1}, \mathrm{a}_{2} \mathrm{~b}_{2}, \mathrm{a}_{3} \mathrm{~b}_{3}\right)$, where $\mathrm{a}_{1}, \mathrm{a}_{2}, \mathrm{a}_{3} ; \mathrm{b}_{1}, \mathrm{~b}_{2}, \mathrm{~b}_{3}$ are all non-zero positive real numbers.

(iv) Division: $\frac{\widetilde{A}}{\widetilde{\mathrm{B}}}=\left(\frac{\mathrm{a}_{1}}{\mathrm{~b}_{3}}, \frac{\mathrm{a}_{2}}{\mathrm{~b}_{2}}, \frac{\mathrm{a}_{3}}{\mathrm{~b}_{1}}\right)$, where $\mathrm{b}_{1}, \mathrm{~b}_{2}, \mathrm{~b}_{3}$ are all non-zero positive real numbers.

(v) Scalar Multiplication: For any real number K,

$$
\begin{aligned}
& K \widetilde{A}=\left(K a_{1}, K a_{2}, K a_{3}\right), \text { Where } K \geq 0, \\
& K \widetilde{A}=\left(K a_{3}, K a_{2}, K a_{1}\right) \text { Where } K<0,
\end{aligned}
$$

Definition 2.7: The $\alpha$ - cut of $\widetilde{A}$ is defined by $A_{\alpha}=\left\{x: \mu_{\widetilde{A}}(x)=\alpha, \alpha \geq 0\right\}$.

Definition 2.8: Among the various shapes of fuzzy number, triangular fuzzy number (TFN) is the most popular one. $\widetilde{A}$ is represented by the triplet $\left(a_{1}, a_{2}, a_{3}\right)$ and is defined by its continuous membership function where $\mu_{\widetilde{A}}(x): X \rightarrow[0,1]$ is given by

$$
\mu_{\widetilde{A}}(x)=f(x)=\left\{\begin{array}{cl}
1-\frac{a_{2}-x}{a_{2}-a_{1}}, & \text { for } a_{1} \leq x \leq a_{2} \\
1, & \text { for } x=a_{2} \\
1-\frac{x-a_{1}}{a_{2}-a_{1}}, & \text { for } a_{2} \leq x \leq a_{3} \\
0, & \text { for Otherwise }
\end{array}\right.
$$

Definition 2.9: The $\alpha$-level set of the triangular number $\widetilde{A}=\left(a_{1}, a_{2}, a_{3}\right)$ is :

$$
A_{\alpha}=\left\{x: \mu_{\widetilde{A}}(x) \geq \alpha\right\}=\left[A_{L}(\alpha), A_{R}(\alpha)\right] .
$$

Where $A_{L}(\alpha)=a_{1}+\left(a_{2}-a_{1}\right) \alpha, \alpha \in[0,1]$, And $A_{R}(\alpha)=a_{3}-\left(a_{3}-a_{2}\right) \alpha, \alpha \in[0,1]$.

We represent $\widetilde{A}=\left(a_{1}, a_{2}, a_{3}\right)=\cup\left[A_{L}(\alpha), A_{R}(\alpha)\right] ; 0 \leq \alpha \leq 1$.

Definition 2.10: Defuzzification of $\widetilde{A}$ can be found by Signed Distance Method. If $\widetilde{A}$ is a triangular fuzzy number then sign distance from $\widetilde{A}$ to 0 is defined as:

$$
d(\widetilde{A}, 0)=\frac{1}{2} \int_{0}^{1}\left[\left\{A_{L}(\alpha), A_{R}(\alpha)\right\}, 0\right] d \alpha
$$


Where, $A_{\alpha}=\left[A_{L}(\alpha), A_{R}(\alpha)\right]$ and $A_{\alpha}=\left[a_{1}+\left(a_{2}-a_{1}\right) \alpha, a_{3}-\left(a_{3}-a_{2}\right) \alpha\right], \alpha \in$ $[0,1]$ is $\alpha$ - cut off fuzzy set $\widetilde{\mathrm{A}}$, which is a close interval.

\section{NOTATIONS AND ASSUMPTIONS:}

This inventory model is produced based on the accompanying Assumptions and Notations which are utilized all through this paper in Crisp and Fuzzy Environment.

\section{Notations:}

$\mathrm{I}(\mathrm{t})$ : The inventory level at any time $\mathrm{t}, \mathrm{t} \geq 0$.

$\mathrm{C}_{1}$ : The fixed operating cost of the inventory.

$>\mathrm{C}_{2}:$ The advertisement cost per advertisement.

lp : The production cost per unit per unit time.

Tac: The total average cost per unit per cycle.

$\widetilde{\mathrm{C}_{1}}$ : The Fuzzy fixed operating cost of the inventory.

$>\widetilde{\mathrm{C}_{2}}$ : Fuzzy advertisement cost per advertisement.

$>$ Tac: Fuzzy total average cost per unit per cycle.

$>t_{1}$ : The production time when the quality of products in stock reaches maximum $\mathrm{L}_{\mathrm{m}}, \mathrm{t}_{1}>0$.

$>t_{2}$ : The time duration where there is no production but deteriorating and end of $t_{2}$ the inventory level diminished gradually to zero, $t_{2}>0$.

$>t_{1}+t_{2}:$ The length of cycle time, $t_{1}+t_{2}>0$.

\section{* Assumptions :}

$>$ The rate of non-instantaneous decay whenever any time $t>0$ is time proportional, $\theta(t)=\beta t$; where, $\beta(0<\beta<1)$ is the scale parameter.

$>$ The demand rate $\mathrm{D}(\mathrm{m}, \mathrm{p}, \mathrm{f})=\frac{\mathrm{mf}}{\mathrm{p}}$ is dependent on population $(\mathrm{m})$, selling price (p) of an item and the frequency of advertisement (f), where $m, p, f>0$.

$>$ Production rate $\mathrm{K}(\mathrm{k}, \mathrm{m}, \mathrm{p}, \mathrm{a})=\mathrm{kD}(\mathrm{m}, \mathrm{p}, \mathrm{f})=\mathrm{k} \frac{\mathrm{mf}}{\mathrm{p}}$, where $\mathrm{k}$ is a positive constant.

Holding cost is $h$, a constant. 
INDEPENDENT JOURNAL OF MANAGEMENT \& PRODUCTION (IJM\&P)

http://www.ijmp.jor.br

V. 10, n. 5, September-October 2019

ISSN: 2236-269X

DOI: 10.14807/ijmp.v10i5.897

$>$ Lead time is zero or negligible.

$>$ The discounted rate $\mathrm{d}$ per unit per unit time.

$>$ The Defective items rate $r$ per time for each cycle.

> The horizontal planning takes place at an infinite rate.

There is no replenishment or repair of deteriorating and defective items takes place in the given cycle.

$>$ The lead time is considered zero.

\section{PRODUCTION INVENTORY MODEL IN CRISP ENVIRONMENT IS} PRODUCED AS FOLLOW:

Let, the producer start to produce items at the start of each cycle when $t=0$ to satisfy the arriving demands in the inventory system. At the end of time $t_{1}$, the production stopped where number of $r$ items are produced defective. We assume the inventory level reached to its highest level $L_{m}(>0)$ at end of $t_{1}$. During the time interval $t_{2}$, the inventory level diminishes owing to customer demand and deterioration and finally falls to zero at $t=t_{1}+t_{2}$. Figure -2 delineates the inventory level of the proposed model.

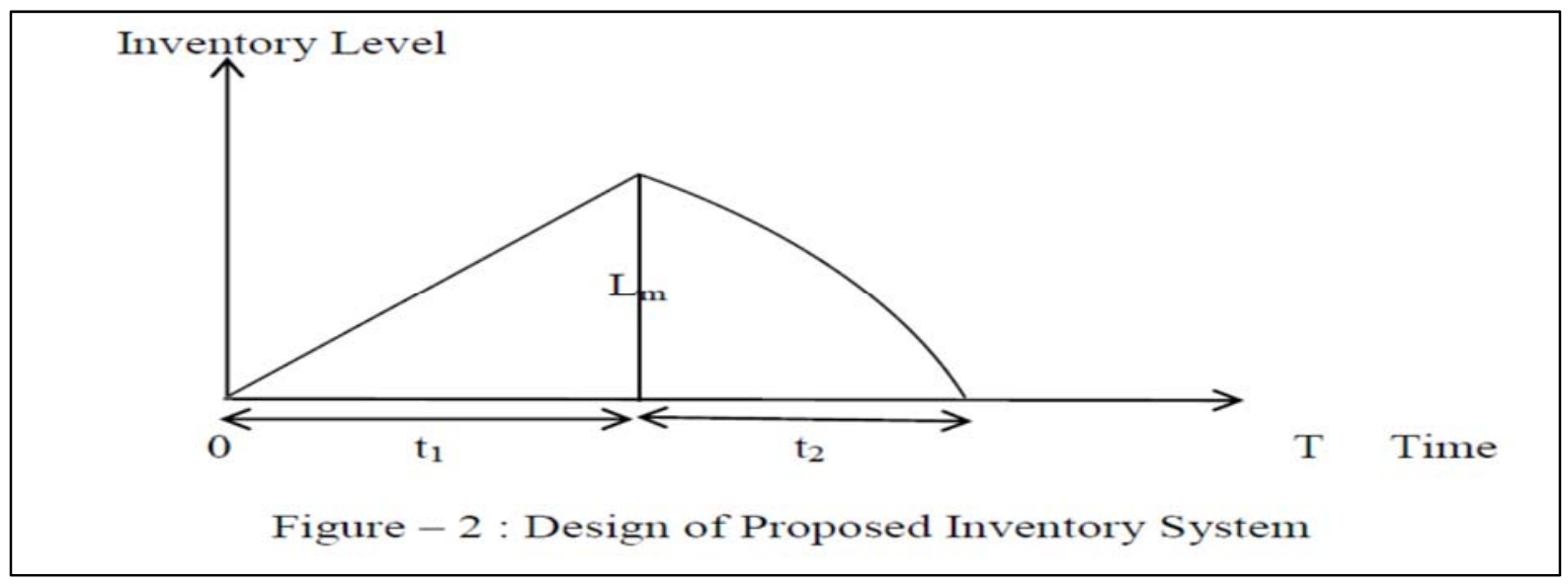

Figure 2: Design of Proposed Inventory System

The Inventory Level in $t_{1}\left(0 \leq t \leq t_{1}\right)$ : The produced items during $t_{1}$ would be depleted due to the instant demand as well as defective items. Under above assumption, during the period $t_{1}$, the inventory status of the system is given by the following differential equation-

$$
\frac{\mathrm{dI}_{1}(\mathrm{t})}{\mathrm{dt}}=\mathrm{kD}(\mathrm{m}, \mathrm{p}, \mathrm{f})-\mathrm{D}(\mathrm{m}, \mathrm{p}, \mathrm{f})-\mathrm{r}, \text { for }\left(0 \leq t \leq t_{1}\right)
$$


From the initial Condition $\mathrm{I}_{1}(0)=0$ and $\mathrm{I}_{1}\left(\mathrm{t}_{1}\right)=\mathrm{L}_{\mathrm{m}}$ get from above equation (1),

$$
\mathrm{I}_{1}(\mathrm{t})=\left(\mathrm{k} \frac{\mathrm{mf}}{\mathrm{p}}-\frac{\mathrm{mf}}{\mathrm{p}}-\mathrm{r}\right) \mathrm{t}, \quad \text { for }\left(0 \leq t \leq t_{1}\right)
$$

And $\quad \mathrm{L}_{\mathrm{m}}=\left(\mathrm{k} \frac{\mathrm{mf}}{\mathrm{p}}-\frac{\mathrm{mf}}{\mathrm{p}}-\mathrm{r}\right) \mathrm{t}_{1}$,

The Inventory Level in $t_{2}\left(t_{2} \leq t \leq t_{1}+t_{2}\right)$ : In this time, the inventory declines due to customers' demand and deterioration. Hence, the status of the inventory level during $t_{2}$ is governed by the following Differential Equation,

$$
\frac{\mathrm{dI}_{2}(\mathrm{t})}{\mathrm{dt}}+\beta \mathrm{I}_{2}(\mathrm{t})=-\mathrm{D}(\mathrm{m}, \mathrm{p}, \mathrm{f}), \text { for }\left(\mathrm{t}_{1} \leq \mathrm{t} \leq \mathrm{t}_{1}+\mathrm{t}_{2}\right)
$$

From the boundary condition $I_{2}\left(t_{1}+t_{2}\right)=0$ and dismissing the higher intensity of $\beta$ and taking taking initial two terms of the exponential series, we get,

$$
I_{2}(t)=\left[\frac{m f}{p}\right]\left[t_{1}+t_{2}-t+\beta \frac{t^{3}}{3}+\frac{\beta\left(t_{1}+t_{2}\right)^{3}}{6}-\beta\left(t_{1}+t_{2}\right) \frac{t^{2}}{2}\right],
$$

According to above discussion, the following cost function can be derived.

1. The Operating cost during the period $\left[0, t_{1}+t_{2}\right]: C_{1}$

2. The Production cost during the period $\left[0, t_{1}\right]: \operatorname{lpk} \frac{m f}{p} t_{1}=k \operatorname{lmf} t_{1}$,

3. The Inventory Holding Cost during the period $\left[0, t_{1}+t_{2}\right]: \int_{0}^{t_{1}} h I_{1}(t) d t+$ $\int_{t_{1}}^{t_{1}+t_{2}} h I_{2}(t) d t$

Using equation (2) and (5), then integrating, we get from above the Holding Cost,

$h\left(k \frac{m f}{p}-\frac{m f}{p}-r\right) \frac{t_{1}^{2}}{2}+h \frac{m f}{p}\left\{\frac{\left(t_{1}+t_{2}\right)^{2}}{2}+\beta \frac{\left(t_{1}+t_{2}\right)^{4}}{12}-t_{1} t_{2}-\frac{t_{1}{ }^{2}}{2}-\beta \frac{t_{1}{ }^{4}}{12}-\frac{\beta\left(t_{1}+t_{2}\right)^{3}}{6} t_{1}+\right.$ $\left.\beta\left(t_{1}+t_{2}\right) \frac{t_{1}{ }^{3}}{6}\right\}$

4. The Deteriorating Cost during the period $\left[t_{1}, t_{1}+t_{2}\right]: \operatorname{lp} \int_{t_{1}}^{t_{1}+t_{2}} \beta t_{2}(t) d t$

Using equation (5) and integrating, we get from above the Deteriorating Cost,

$$
\beta \operatorname{lmf}\left[\frac{\left(\mathrm{t}_{1}+\mathrm{t}_{2}\right)^{3}}{6}-\frac{\mathrm{t}_{1}^{3}}{6}-\frac{\mathrm{t}_{1}^{2}}{2} \mathrm{t}_{2}\right]
$$

5. The Advertisement cost during the period $\left[0, t_{1}+t_{2}\right]: C_{2} f$

6. The Price Discount during the period $\left[t_{1}, t_{1}+t_{2}\right]: \operatorname{lpd} \int_{t_{1}}^{t_{1}+t_{2}} \frac{m f}{p} d t$ 
We get from above; Price Discount is ldmft $_{2}$

Therefore the total average cost function per cycle: $\frac{1}{\left(\mathrm{t}_{1}+\mathrm{t}_{2}\right)}$ [Operating Cost + Production Cost + Inventory Holding Cost + Deteriorating Cost + Advertisement Cost + Price Discount].

Hence the average net cost function is

$$
\begin{array}{r}
\operatorname{Tac}\left(\mathrm{t}_{1}, \mathrm{t}_{2}\right)=\frac{1}{\left(\mathrm{t}_{1}+\mathrm{t}_{2}\right)}\left[\mathrm{C}_{1}+\mathrm{klmft} \mathrm{t}_{1}+\mathrm{h}\left(\mathrm{k} \frac{\mathrm{mf}}{\mathrm{p}}-\frac{\mathrm{mf}}{\mathrm{p}}-\mathrm{r}\right) \frac{\mathrm{t}_{1}^{2}}{2}+\mathrm{h} \frac{\mathrm{mf}}{\mathrm{p}}\left\{\frac{\left(\mathrm{t}_{1}+\mathrm{t}_{2}\right)^{2}}{2}+\beta \frac{\left(\mathrm{t}_{1}+\mathrm{t}_{2}\right)^{4}}{12}-\right.\right. \\
\left.\left.\mathrm{t}_{1} \mathrm{t}_{2}-\frac{\mathrm{t}_{1}{ }^{2}}{2}-\beta \frac{\mathrm{t}_{1}{ }^{4}}{12}-\frac{\beta\left(\mathrm{t}_{1}+\mathrm{t}_{2}\right)^{3}}{6} \mathrm{t}_{1}+\beta\left(\mathrm{t}_{1}+\mathrm{t}_{2}\right) \frac{\mathrm{t}_{1}{ }^{3}}{6}\right\}+\beta \operatorname{lmf}\left[\frac{\left(\mathrm{t}_{1}+\mathrm{t}_{2}\right)^{3}}{6}-\frac{\mathrm{t}_{1}^{3}}{6}-\frac{\mathrm{t}_{1}^{2}}{2} \mathrm{t}_{2}\right]+\mathrm{C}_{2} \mathrm{f}+1 \mathrm{dmft}_{2}\right],
\end{array}
$$

Now, the necessary condition for the total average cost function of the system is minimize if equation (12) is satisfy,

$$
\begin{aligned}
& \frac{\partial \operatorname{Tac}\left(t_{1}, t_{2}\right)}{\partial t_{1}}=0, \\
& \text { And } \frac{\partial \operatorname{Tac}\left(t_{1}, t_{2}\right)}{\partial t_{2}}=0,
\end{aligned}
$$

The solution, which might be called feasible solution of the problem, of the conditions (13) and (14) give the optimal solutions of $t_{1}=t_{1}^{*}$ and $t_{2}=t_{2}^{*}$ which minimize $\operatorname{Tac}\left(t_{1}, t_{2}\right)=\operatorname{Tac}\left(t_{1}, t_{2}\right) *$ provide they satisfy the sufficient conditions-

$$
\begin{aligned}
& \frac{\partial^{2} \operatorname{Tac}\left(t_{1}, t_{2}\right)}{\partial t_{1}^{2}} \cdot \frac{\partial^{2} \operatorname{Tac}\left(t_{1}, t_{2}\right)}{\partial t_{2}{ }^{2}}-\left(\frac{\partial^{2} T a c\left(t_{1}, t_{2}\right.}{\partial t_{1} \partial t_{2}}\right)^{2}>0, \\
& \text { And } \frac{\partial^{2} \operatorname{Tac}\left(t_{1}, t_{2}\right)}{\partial t_{1}^{2}}>0 \text { or, } \frac{\partial^{2} T a c\left(t_{1}, t_{2}\right)}{\partial t_{2}{ }^{2}}>0,
\end{aligned}
$$

However, it's difficult to solve the problem by inferring an explicit equation of the solutions from conditions (13) and (14). Therefore, we solve the optimal service level of $t_{1}=t_{1}^{*}$ and $t_{2}=t_{2}^{*}$ by using the software LINGO 17.0. Moreover, we also verify that the sufficient conditions of the optimality of the solutions of $t_{1}=t_{1}^{*}$ and $t_{2}=$ $t_{2}^{*}$ are satisfied (i.e. inequalities (15) and (16)) under certain conditions.

\section{THE PROPOSED INVENTORY MODEL IN FUZZY ENVIRONMENT IS PRODUCED AS FOLLOW:}

Presently the above model will be produced in fuzzy Environment. Due to uncertainly, it is difficult to characterize every one of the parameters definitely. Let us 
INDEPENDENT JOURNAL OF MANAGEMENT \& PRODUCTION (IJM\&P)

http://Www.ijmp.jor.br

V. 10, n. 5, September-October 2019

ISSN: 2236-269X

DOI: 10.14807/ijmp.v10i5.897

assume that, $\widetilde{\mathrm{C}_{1}}=\left(\mathrm{C}_{1}^{1}, \mathrm{C}_{1}^{2}, \mathrm{C}_{1}^{3}\right), \tilde{\mathrm{h}}=\left(\mathrm{h}^{1}, \mathrm{~h}^{2}, \mathrm{~h}^{3}\right), \quad \tilde{\mathrm{p}}=\left(\mathrm{p}^{1}, \mathrm{p}^{2}, \mathrm{p}^{3}\right), \widetilde{\mathrm{C}_{2}}=\left(\mathrm{C}_{2}^{1}, \mathrm{C}_{2}^{2}, \mathrm{C}_{2}^{3}\right)$, be Triangular Fuzzy Number in LR-form then the total average cost function of the system per unit time in fuzzy environment is given by-

$\left.\operatorname{Tac} \widetilde{\left(t_{1}\right.}, t_{2}\right)=\frac{1}{\left(t_{1}+t_{2}\right)}\left[\widetilde{\mathrm{C}_{1}}+k l m f t_{1}+\tilde{h}\left(k \frac{m f}{\widetilde{p}}-\frac{m f}{\widetilde{p}}-r\right) \frac{t_{1}^{2}}{2}+\tilde{h} \frac{m f}{\widetilde{p}}\left\{\frac{\left(t_{1}+t_{2}\right)^{2}}{2}+\beta \frac{\left(t_{1}+t_{2}\right)^{4}}{12}-t_{1} t_{2}-\right.\right.$ $\left.\left.\frac{\mathrm{t}_{1}{ }^{2}}{2}-\beta \frac{\mathrm{t}_{1}{ }^{4}}{12}-\frac{\beta\left(\mathrm{t}_{1}+\mathrm{t}_{2}\right)^{3}}{6} \mathrm{t}_{1}+\beta\left(\mathrm{t}_{1}+\mathrm{t}_{2}\right) \frac{\mathrm{t}_{1}{ }^{3}}{6}\right\}+\beta \operatorname{lmf}\left\{\frac{\left(\mathrm{t}_{1}+\mathrm{t}_{2}\right)^{3}}{6}-\frac{\mathrm{t}_{1}^{3}}{6}-\frac{\mathrm{t}_{1}^{2}}{2} \mathrm{t}_{2}\right\}+\widetilde{\mathrm{C}_{2}} \mathrm{f}+\mathrm{ddmft}_{2}\right]$ Or, Tac $\left.\widetilde{t_{1}}, t_{2}\right)=\frac{1}{\left(t_{1}+t_{2}\right)}\left[\left(C_{1}^{1}, C_{1}^{2}, C_{1}^{3}\right)+k_{m f t}+\left(h^{1}, h^{2}, h^{3}\right)\left(k \frac{m f}{\left(p^{1}, p^{2}, p^{3}\right)}-\frac{m f}{\left(p^{1}, p^{2}, p^{3}\right)}-r\right) \frac{t_{1}^{2}}{2}\right.$ $+\left(h^{1}, h^{2}, h^{3}\right) \frac{m f}{\left(p^{1}, p^{2}, p^{3}\right)}\left\{\frac{\left(t_{1}+t_{2}\right)^{2}}{2}+\beta \frac{\left(t_{1}+t_{2}\right)^{4}}{12}-t_{1} t_{2}-\frac{t_{1}{ }^{2}}{2}-\beta \frac{t_{1}{ }^{4}}{12}-\frac{\beta\left(t_{1}+t_{2}\right)^{3}}{6} t_{1}+\beta\left(t_{1}+t_{2}\right) \frac{t_{1}{ }^{3}}{6}\right\}$ $\left.+\beta \operatorname{lmf}\left\{\frac{\left(\mathrm{t}_{1}+\mathrm{t}_{2}\right)^{3}}{6}-\frac{\mathrm{t}_{1}^{3}}{6}-\frac{\mathrm{t}_{1}^{2}}{2} \mathrm{t}_{2}\right\}+\left(\mathrm{C}_{2}^{1}, \mathrm{C}_{2}^{2}, \mathrm{C}_{2}^{3}\right) \mathrm{f}+\operatorname{ldmft}_{2}\right]=(\mathrm{U}, \mathrm{V}, \mathrm{W})$ (Say)

Where, $U=\frac{1}{\left(t_{1}+t_{2}\right)}\left[C_{1}^{1}+k^{2} \operatorname{lmft}_{1}+h^{1}\left(k \frac{m f}{p^{1}}-\frac{m f}{p^{1}}-r\right) \frac{t_{1}^{2}}{2}+h^{1} \frac{m f}{p^{1}}\left\{\frac{\left(t_{1}+t_{2}\right)^{2}}{2}+\beta \frac{\left(t_{1}+t_{2}\right)^{4}}{12}-t_{1} t_{2}-\right.\right.$ $\left.\left.\frac{\mathrm{t}_{1}{ }^{2}}{2}-\beta \frac{\mathrm{t}_{1}{ }^{4}}{12}-\frac{\beta\left(\mathrm{t}_{1}+\mathrm{t}_{2}\right)^{3}}{6} \mathrm{t}_{1}+\beta\left(\mathrm{t}_{1}+\mathrm{t}_{2}\right) \frac{\mathrm{t}_{1}{ }^{3}}{6}\right\}+\beta \operatorname{lmf}\left\{\frac{\left(\mathrm{t}_{1}+\mathrm{t}_{2}\right)^{3}}{6}-\frac{\mathrm{t}_{1}^{3}}{6}-\frac{\mathrm{t}_{1}^{2}}{2} \mathrm{t}_{2}\right\}+\mathrm{C}_{2}^{1} \mathrm{f}+\operatorname{ldmft}_{2}\right] ;$

$V=\frac{1}{\left(t_{1}+t_{2}\right)}\left[C_{1}^{2}+k l m f t_{1}+h^{2}\left(k \frac{m f}{p^{2}}-\frac{m f}{p^{2}}-r\right) \frac{t_{1}^{2}}{2}+h^{2} \frac{m f}{p^{2}}\left\{\frac{\left(t_{1}+t_{2}\right)^{2}}{2}+\beta \frac{\left(t_{1}+t_{2}\right)^{4}}{12}-t_{1} t_{2}-\frac{t_{1}{ }^{2}}{2}-\right.\right.$ $\left.\left.\beta \frac{\mathrm{t}^{4}{ }^{4}}{12}-\frac{\beta\left(\mathrm{t}_{1}+\mathrm{t}_{2}\right)^{3}}{6} \mathrm{t}_{1}+\beta\left(\mathrm{t}_{1}+\mathrm{t}_{2}\right) \frac{\mathrm{t}_{1}{ }^{3}}{6}\right\}+\beta \operatorname{lmf}\left\{\frac{\left(\mathrm{t}_{1}+\mathrm{t}_{2}\right)^{3}}{6}-\frac{\mathrm{t}_{1}^{3}}{6}-\frac{\mathrm{t}_{1}^{2}}{2} \mathrm{t}_{2}\right\}+C_{2}^{2} \mathrm{f}+1 \mathrm{dmft} \mathrm{t}_{2}\right] ;$

And, $W=\frac{1}{\left(t_{1}+t_{2}\right)}\left[C_{1}^{3}+k_{m f t}+h^{3}\left(k \frac{m f}{p^{3}}-\frac{m f}{p^{3}}-r\right) \frac{t_{1}^{2}}{2}+h^{3} \frac{m f}{p^{3}}\left\{\frac{\left(t_{1}+t_{2}\right)^{2}}{2}+\beta \frac{\left(t_{1}+t_{2}\right)^{4}}{12}-t_{1} t_{2}-\right.\right.$ $\left.\left.\frac{\mathrm{t}_{1}{ }^{2}}{2}-\beta \frac{\mathrm{t}_{1}{ }^{4}}{12}-\frac{\beta\left(\mathrm{t}_{1}+\mathrm{t}_{2}\right)^{3}}{6} \mathrm{t}_{1}+\beta\left(\mathrm{t}_{1}+\mathrm{t}_{2}\right) \frac{\mathrm{t}_{1}{ }^{3}}{6}\right\}+\beta \operatorname{lmf}\left\{\frac{\left(\mathrm{t}_{1}+\mathrm{t}_{2}\right)^{3}}{6}-\frac{\mathrm{t}_{1}^{3}}{6}-\frac{\mathrm{t}_{1}^{2}}{2} \mathrm{t}_{2}\right\}+\mathrm{C}_{2}^{3} \mathrm{f}+\operatorname{ldmft} \mathrm{t}_{2}\right]$

The $\alpha$-cuts, $A_{L}(\alpha)$ and $A_{R}(\alpha)$ of triangular fuzzy number Tac $\left.\widetilde{\left(t_{1},\right.} t_{2}\right)$ are given by-

$\mathrm{A}_{\mathrm{L}}(\alpha)=\mathrm{U}+(\mathrm{V}-\mathrm{U}) \alpha=\frac{1}{\left(\mathrm{t}_{1}+\mathrm{t}_{2}\right)}\left[\mathrm{C}_{1}^{1}+\mathrm{klmft}_{1}+\mathrm{h}^{1}\left(\mathrm{k} \frac{\mathrm{mf}}{\mathrm{p}^{1}}-\frac{\mathrm{mf}}{\mathrm{p}^{1}}-\mathrm{r}\right) \frac{\mathrm{t}_{1}^{2}}{2}+\mathrm{h}^{1} \frac{\mathrm{mf}}{\mathrm{p}^{1}}\left\{\frac{\left(\mathrm{t}_{1}+\mathrm{t}_{2}\right)^{2}}{2}+\right.\right.$ $\left.\beta \frac{\left(t_{1}+t_{2}\right)^{4}}{12}-t_{1} t_{2}-\frac{t_{1}{ }^{2}}{2}-\beta \frac{t_{1}{ }^{4}}{12}-\frac{\beta\left(t_{1}+t_{2}\right)^{3}}{6} t_{1}+\beta\left(t_{1}+t_{2}\right) \frac{t_{1}{ }^{3}}{6}\right\}+\beta \operatorname{lmf}\left\{\frac{\left(t_{1}+t_{2}\right)^{3}}{6}-\frac{t_{1}^{3}}{6}-\frac{t_{1}^{2}}{2} t_{2}\right\}+$ $\left.\mathrm{C}_{2}^{1} \mathrm{f}+\mathrm{ldmft}_{2}\right]+\frac{1}{\left(\mathrm{t}_{1}+\mathrm{t}_{2}\right)}\left[\left(\mathrm{C}_{1}^{2}-\mathrm{C}_{1}^{1}\right)+\mathrm{h}^{2}\left(\mathrm{k} \frac{\mathrm{mf}}{\mathrm{p}^{2}}-\frac{\mathrm{mf}}{\mathrm{p}^{2}}-\mathrm{r}\right) \frac{\mathrm{t}_{1}^{2}}{2}-\mathrm{h}^{1}\left(\mathrm{k} \frac{\mathrm{mf}}{\mathrm{p}^{1}}-\frac{\mathrm{mf}}{\mathrm{p}^{1}}-\mathrm{r}\right) \frac{\mathrm{t}_{1}^{2}}{2}+\left(\mathrm{h}^{2} \frac{\mathrm{mf}}{\mathrm{p}^{2}}-\right.\right.$ $\left.\mathrm{h}^{1} \frac{\mathrm{mf}}{\mathrm{p}^{1}}\right) \quad\left\{\frac{\left(\mathrm{t}_{1}+\mathrm{t}_{2}\right)^{2}}{2}+\beta \frac{\left(\mathrm{t}_{1}+\mathrm{t}_{2}\right)^{4}}{12}-\mathrm{t}_{1} \mathrm{t}_{2}-\frac{\mathrm{t}_{1}{ }^{2}}{2}-\beta \frac{\mathrm{t}_{1}{ }^{4}}{12}-\frac{\beta\left(\mathrm{t}_{1}+\mathrm{t}_{2}\right)^{3}}{6} \mathrm{t}_{1}+\beta\left(\mathrm{t}_{1}+\mathrm{t}_{2}\right) \frac{\mathrm{t}_{1}{ }^{3}}{6}\right\}+\quad\left(\mathrm{C}_{2}^{2}-\right.$ $\left.\left.\mathrm{C}_{2}^{1}\right) \mathrm{f}\right] \alpha$

And $\quad \mathrm{A}_{\mathrm{R}}(\alpha)=\mathrm{W}-(\mathrm{W}-\mathrm{V}) \alpha=\frac{1}{\left(\mathrm{t}_{1}+\mathrm{t}_{2}\right)}\left[\mathrm{C}_{1}^{3}+\mathrm{klmft}_{1}+\mathrm{h}^{3}\left(\mathrm{k} \frac{\mathrm{mf}}{\mathrm{p}^{3}}-\frac{\mathrm{mf}}{\mathrm{p}^{3}}-\mathrm{r}\right) \frac{\mathrm{t}_{1}^{2}}{2}+\right.$ $h^{3} \frac{m f}{p^{3}}\left\{\frac{\left(t_{1}+t_{2}\right)^{2}}{2}+\beta \frac{\left(t_{1}+t_{2}\right)^{4}}{12}-t_{1} t_{2}-\frac{t_{1}{ }^{2}}{2}-\beta \frac{t_{1}{ }^{4}}{12}-\frac{\beta\left(t_{1}+t_{2}\right)^{3}}{6} t_{1}+\beta\left(t_{1}+t_{2}\right) \frac{t_{1}{ }^{3}}{6}\right\}+$ 
INDEPENDENT JOURNAL OF MANAGEMENT \& PRODUCTION (IJM\&P)

http://Www.ijmp.jor.br

V. 10, n. 5, September-October 2019

ISSN: 2236-269X

DOI: 10.14807/ijmp.v10i5.897

$\left.\beta \operatorname{lmf}\left\{\frac{\left(\mathrm{t}_{1}+\mathrm{t}_{2}\right)^{3}}{6}-\frac{\mathrm{t}_{1}^{3}}{6}-\frac{\mathrm{t}_{1}^{2}}{2} \mathrm{t}_{2}\right\}+\mathrm{C}_{2}^{3} \mathrm{f}+\mathrm{ldmft}_{2}\right]-\frac{1}{\left(\mathrm{t}_{1}+\mathrm{t}_{2}\right)}\left[\left(\mathrm{C}_{1}^{3}-\mathrm{C}_{1}^{2}\right)+\mathrm{h}^{3}\left(\mathrm{k} \frac{\mathrm{mf}}{\mathrm{p}^{3}}-\frac{\mathrm{mf}}{\mathrm{p}^{3}}-\mathrm{r}\right) \frac{\mathrm{t}_{1}^{2}}{2}\right.$ $-h^{2}\left(k \frac{m f}{p^{2}}-\frac{m f}{p^{2}}-r\right) \frac{t_{1}^{2}}{2}+\left(h^{3} \frac{m f}{p^{3}}-h^{2} \frac{m f}{p^{2}}\right) \quad\left\{\frac{\left(t_{1}+t_{2}\right)^{2}}{2}+\beta \frac{\left(t_{1}+t_{2}\right)^{4}}{12}-t_{1} t_{2}-\frac{t_{1}{ }^{2}}{2}-\beta \frac{t_{1}{ }^{4}}{12}-\right.$ $\left.\left.\frac{\beta\left(t_{1}+t_{2}\right)^{3}}{6} t_{1}+\beta\left(t_{1}+t_{2}\right) \frac{t_{1}^{3}}{6}\right\}+\left(C_{2}^{3}-C_{2}^{2}\right) f\right] \alpha$

We defuzzify the fuzzy average total cost function Tac $\left.\widetilde{\left(t_{1}\right.}, t_{2}\right)$ by Signed Distance Method as follows,

$\left.\operatorname{Tac}_{s d} \widetilde{\left(t_{1}\right.}, t_{2}\right)=\frac{1}{2\left(t_{1}+t_{2}\right)}\left[C_{1}^{1}+\operatorname{klmft}_{1}+h^{1}\left(k \frac{m f}{p^{1}}-\frac{m f}{p^{1}}-r\right) \frac{t_{1}^{2}}{2}+h^{1} \frac{m f}{p^{1}}\left\{\frac{\left(t_{1}+t_{2}\right)^{2}}{2}+\beta \frac{\left(t_{1}+t_{2}\right)^{4}}{12}-\right.\right.$ $\left.\left.\mathrm{t}_{1} \mathrm{t}_{2}-\frac{\mathrm{t}_{1}{ }^{2}}{2}-\beta \frac{\mathrm{t}_{1}{ }^{4}}{12}-\frac{\beta\left(\mathrm{t}_{1}+\mathrm{t}_{2}\right)^{3}}{6} \mathrm{t}_{1}+\beta\left(\mathrm{t}_{1}+\mathrm{t}_{2}\right) \frac{\mathrm{t}_{1}{ }^{3}}{6}\right\}+\beta \operatorname{lmf}\left\{\frac{\left(\mathrm{t}_{1}+\mathrm{t}_{2}\right)^{3}}{6}-\frac{\mathrm{t}_{1}^{3}}{6}-\frac{\mathrm{t}_{1}^{2}}{2} \mathrm{t}_{2}\right\}+C_{2}^{1} \mathrm{f}+1 \mathrm{dmft} \mathrm{t}_{2}\right]$ $+\frac{1}{4\left(\mathrm{t}_{1}+\mathrm{t}_{2}\right)}\left[\left(C_{1}^{2}-C_{1}^{1}\right)+\mathrm{h}^{2}\left(\mathrm{k} \frac{\mathrm{mf}}{\mathrm{p}^{2}}-\frac{\mathrm{mf}}{\mathrm{p}^{2}}-\mathrm{r}\right) \frac{\mathrm{t}_{1}^{2}}{2}-\mathrm{h}^{1}\left(\mathrm{k} \frac{\mathrm{mf}}{\mathrm{p}^{1}}-\frac{\mathrm{mf}}{\mathrm{p}^{1}}-\mathrm{r}\right) \frac{\mathrm{t}_{1}^{2}}{2}+\left(\mathrm{h}^{2} \frac{\mathrm{mf}}{\mathrm{p}^{2}}-\mathrm{h}^{1} \frac{\mathrm{mf}}{\mathrm{p}^{1}}\right)\right.$ $\left\{\frac{\left(t_{1}+t_{2}\right)^{2}}{2}+\beta \frac{\left(t_{1}+t_{2}\right)^{4}}{12}-t_{1} t_{2}-\frac{t_{1}{ }^{2}}{2}-\beta \frac{t_{1}{ }^{4}}{12}-\frac{\beta\left(t_{1}+t_{2}\right)^{3}}{6} t_{1}+\beta\left(t_{1}+t_{2}\right) \frac{t_{1}{ }^{3}}{6}\right\}+\quad\left(C_{2}^{2}-C_{2}^{1}\right) f$ ]$+\frac{1}{2\left(\mathrm{t}_{1}+\mathrm{t}_{2}\right)}\left[\mathrm{C}_{1}^{3}+\mathrm{klmft}_{1}+\mathrm{h}^{3}\left(\mathrm{k} \frac{\mathrm{mf}}{\mathrm{p}^{3}}-\frac{\mathrm{mf}}{\mathrm{p}^{3}}-\mathrm{r}\right) \frac{\mathrm{t}_{1}^{2}}{2}+\mathrm{h}^{3} \frac{\mathrm{mf}}{\mathrm{p}^{3}}\left\{\frac{\left(\mathrm{t}_{1}+\mathrm{t}_{2}\right)^{2}}{2}+\beta \frac{\left(\mathrm{t}_{1}+\mathrm{t}_{2}\right)^{4}}{12}-\mathrm{t}_{1} \mathrm{t}_{2}-\right.\right.$ $\left.\left.\frac{\mathrm{t}_{1}{ }^{2}}{2}-\beta \frac{\mathrm{t}_{1}{ }^{4}}{12}-\frac{\beta\left(\mathrm{t}_{1}+\mathrm{t}_{2}\right)^{3}}{6} \mathrm{t}_{1}+\beta\left(\mathrm{t}_{1}+\mathrm{t}_{2}\right) \frac{\mathrm{t}_{1}{ }^{3}}{6}\right\}+\beta \operatorname{lmf}\left\{\frac{\left(\mathrm{t}_{1}+\mathrm{t}_{2}\right)^{3}}{6}-\frac{\mathrm{t}_{1}^{3}}{6}-\frac{\mathrm{t}_{1}^{2}}{2} \mathrm{t}_{2}\right\}+\mathrm{C}_{2}^{3} \mathrm{f}+\operatorname{ldmft}_{2}\right]-$ $\frac{1}{4\left(\mathrm{t}_{1}+\mathrm{t}_{2}\right)}\left[\left(\mathrm{C}_{1}^{3}-\mathrm{C}_{1}^{2}\right)+\mathrm{h}^{3}\left(\mathrm{k} \frac{\mathrm{mf}}{\mathrm{p}^{3}}-\frac{\mathrm{mf}}{\mathrm{p}^{3}}-\mathrm{r}\right) \frac{\mathrm{t}_{1}^{2}}{2}-\mathrm{h}^{2}\left(\mathrm{k} \frac{\mathrm{mf}}{\mathrm{p}^{2}}-\frac{\mathrm{mf}}{\mathrm{p}^{2}}-\mathrm{r}\right) \frac{\mathrm{t}_{1}^{2}}{2}+\left(\mathrm{h}^{3} \frac{\mathrm{mf}}{\mathrm{p}^{3}}-\mathrm{h}^{2} \frac{\mathrm{mf}}{\mathrm{p}^{2}}\right)\left\{\frac{\left(\mathrm{t}_{1}+\mathrm{t}_{2}\right)^{2}}{2}+\right.\right.$ $\left.\left.\beta \frac{\left(t_{1}+t_{2}\right)^{4}}{12}-t_{1} t_{2}-\frac{t_{1}{ }^{2}}{2}-\beta \frac{t_{1}{ }^{4}}{12}-\frac{\beta\left(t_{1}+t_{2}\right)^{3}}{6} t_{1}+\beta\left(t_{1}+t_{2}\right) \frac{t_{1}{ }^{3}}{6}\right\}+\left(C_{2}^{3}-C_{2}^{2}\right) f\right]$

Now, the necessary condition for the average total cost function of the system is minimize if equation (18) is satisfy,

$$
\frac{\partial \operatorname{Tac}_{s \mathrm{~d}}\left(\mathrm{t}_{1}, \mathrm{t}_{2}\right)}{\partial \mathrm{t}_{1}}=0
$$

And $\quad \frac{\partial \operatorname{Tac}_{s \mathrm{~d}}\left(\mathrm{t}_{1}, \mathrm{t}_{2}\right)}{\partial \mathrm{t}_{2}}=0$

The solution, which might be called feasible solution of the problem, of the conditions (19) and (20) give the optimal solutions of $t_{1}=t_{1}^{*}$ and $t_{2}=t_{2}^{*}$ which minimize $\operatorname{Tac}_{s d}\left(t_{1}, t_{2}\right)=\operatorname{Tac}_{s d}\left(t_{1}, t_{2}\right)^{*}$ provide they satisfy the sufficient conditions-

$$
\begin{aligned}
& \frac{\left.\partial^{2} \operatorname{Tac}_{\mathrm{sd}} \overline{\left(\mathrm{t}_{1}\right.}, \mathrm{t}_{2}\right)}{\partial \mathrm{t}_{1}^{2}} \cdot \frac{\partial^{2} \mathrm{Tac}_{\mathrm{sd}} \overline{\left(\mathrm{t}_{1}, \mathrm{t}_{2}\right)}}{\partial \mathrm{t}_{2}^{2}}-\left(\frac{\partial^{2} \mathrm{Tac}_{\mathrm{sd}} \overline{\left(\mathrm{t}_{1}, \mathrm{t}_{2}\right)}}{\partial \mathrm{t}_{1} \partial \mathrm{t}_{2}}\right)^{2}>0 \\
& \text { And } \quad \frac{\left.\partial^{2} \mathrm{Tac}_{s \overline{\mathrm{d}}\left(\mathrm{t}_{1}\right.}, \mathrm{t}_{2}\right)}{\partial \mathrm{t}_{1}^{2}}>0 \quad \text { Or, } \quad \frac{\left.\partial^{2} \mathrm{Tac}_{\mathrm{sd}} \overline{\left(\mathrm{t}_{1}\right.}, \mathrm{t}_{2}\right)}{\partial \mathrm{t}_{2}^{2}}>0
\end{aligned}
$$


INDEPENDENT JOURNAL OF MANAGEMENT \& PRODUCTION (IJM\&P)

http://Www.ijmp.jor.br

V. 10, n. 5, September-October 2019

ISSN: 2236-269X

DOI: 10.14807/ijmp.v10i5.897

However, it's difficult to solve the problem by inferring an explicit equation of the solutions from conditions (19) and (20). Therefore, we solve the optimal service level $t_{1}^{*}$ and the optimal cycle time $t_{1}^{*}+t_{2}^{*}$ by using the software LINGO 16.0. Moreover, we also verify that the sufficient conditions of the optimality of the solutions $t_{1}^{*}$ and $t_{2}^{*}$ are satisfied (i.e. inequalities (21) and (22)) under certain conditions.

Similarly, the highest inventory level per unit time in fuzzy environment is given by

$$
\widetilde{\mathrm{L}_{\mathrm{m}}}=\left(\mathrm{k} \frac{\mathrm{mf}}{\widetilde{\mathrm{p}}}-\frac{\mathrm{mf}}{\widetilde{\mathrm{p}}}-\mathrm{r}\right) \mathrm{t}_{1}=\left(\mathrm{k} \frac{\mathrm{mf}}{(\mathrm{p} 1, \mathrm{p} 2, \mathrm{p} 3)}-\frac{\mathrm{mf}}{(\mathrm{p} 1, \mathrm{p} 2, \mathrm{p} 3)}-\mathrm{r}\right) \mathrm{t}_{1}
$$

Defuzzified value of fuzzy number $\widetilde{\mathrm{L}_{\mathrm{m}}}$ by using Signed Distance Method is given by-

$$
\begin{gathered}
\left(\widetilde{\mathrm{L}_{\mathrm{m}}}\right)_{\mathrm{sd}}=\frac{1}{2}\left(\mathrm{k} \frac{\mathrm{mf}}{\mathrm{p} 1}-\frac{\mathrm{mf}}{\mathrm{p} 1}-\mathrm{r}\right) \mathrm{t}_{1}+\frac{1}{4}\left(\left(\mathrm{k} \frac{\mathrm{mf}}{\mathrm{p} 2}-\frac{\mathrm{mf}}{\mathrm{p} 2}-\mathrm{r}\right)-\left(\mathrm{k} \frac{\mathrm{mf}}{\mathrm{p} 1}-\frac{\mathrm{mf}}{\mathrm{p} 1}-\mathrm{r}\right)\right) \mathrm{t}_{1}+\frac{1}{2}\left(\mathrm{k} \frac{\mathrm{mf}}{\mathrm{p} 3}-\right. \\
\left.\frac{\mathrm{mf}}{\mathrm{p} 3}-\mathrm{r}\right) \mathrm{t}_{1}-\frac{1}{4}\left(\left(\mathrm{k} \frac{\mathrm{mf}}{\mathrm{p} 3}-\frac{\mathrm{mf}}{\mathrm{p} 3}-\mathrm{r}\right)-\left(\mathrm{k} \frac{\mathrm{mf}}{\mathrm{p} 2}-\frac{\mathrm{mf}}{\mathrm{p} 2}-\mathrm{r}\right)\right) \mathrm{t}_{1}
\end{gathered}
$$

\section{NUMERICAL SOLUTION:}

VI (A): A company produces cell-phones. The company wants to minimize the total expenditure. The demand of cell-phones dependent on population $(m=2565)$ of that area, selling price $(p)$ which is near about \$. 11.6, never less than \$ 8.6 and above $\$$ 14.6. and the frequency of advertisement $(f)$ is near about 6 . Production rate is proportional ( $k=1.5$ times) to demand as well as production cost ( $l=0.15$ times) is proportional to selling price. At this situation, the fixed operating cost $\left(C_{1}\right)$ of the inventory system is near about $\$ 175$, never less than $\$ 150$ and above $\$ 200$. Similarly, the holding cost $(h)$ of the inventory system is near about $\$ 1.2$, never less than $\$ 0.84$ and above $\$ 1.56$ and the advertisement cost $\left(C_{2}\right)$ of the inventory system is near about $\$ 50$, never less than $\$ 25$ and above $\$ 75$. The defective cell-phones per unit time are $(r=) 2$ cell-phones where price discount is avail $\$(d=) 1.3$. Also it is observe that the deteriorating rate is $(\beta=) 0.01$. Determine the Optimal value of $t_{1}$, Optimal value of $t_{2}$, optimal total average costs $\operatorname{Tac}\left(t_{1}, t_{2}\right)$ and Optimal value of $L_{m}$.

According to above input data, the solution of the crisp-model is furnishing bellow in table-1. 
Table 1: Output Result in crisp environment

\begin{tabular}{|c|c|c|c|}
\hline $\mathrm{t}_{1}^{*}$ & $\mathrm{t}_{2}^{*}$ & $\operatorname{Tac}\left(\mathrm{t}_{1}, \mathrm{t}_{2}\right)^{*}$ & $\mathrm{~L}_{\mathrm{m}}{ }^{*}$ \\
\hline 0.9168 & 0.5023 & 3816.822 & 484.7281 \\
\hline
\end{tabular}

VI (B): As discussed in the above example if we assume the parameters in fuzzy sense as: $\widetilde{\mathrm{C}_{1}}=(150,175,200), \tilde{\mathrm{p}}=(8.6,11.6,14.6), \widetilde{\mathrm{C}_{2}}=(25,50,75), \widetilde{\mathrm{h}}=$ $(0.84,1.2,1.56)$, where other parameters are unchanged. The solution of fuzzy model by Signed Distance Method is obtained bellow:

(1). When $\widetilde{\mathrm{C}_{1}}, \tilde{\mathrm{p}}, \widetilde{\mathrm{C}_{2}}$ and $\tilde{\mathrm{h}}$ are all Triangular fuzzy numbers then the solution is given bellow table:

Table 2: Output Result in fuzzy environment for case-1.

\begin{tabular}{|c|c|c|c|}
\hline $\mathrm{t}_{1}^{*}$ & $\mathrm{t}_{2}^{*}$ & $\operatorname{Tac}_{\mathrm{sd}}\left(\mathrm{t}_{1}, \mathrm{t}_{2}\right)^{*}$ & $\mathrm{~L}_{\mathrm{m}}{ }^{*}$ \\
\hline 0.9191 & 0.5040 & 3814.925 & 503.4081 \\
\hline
\end{tabular}

(2). When $\widetilde{\mathrm{C}_{1}}, \tilde{\mathrm{p}}$, and $\widetilde{\mathrm{C}_{2}}$ are Triangular fuzzy numbers then the solution is given bellow table:

Table 3: Output Result in fuzzy environment for case-2.

\begin{tabular}{|c|c|c|c|}
\hline $\mathrm{t}_{1}^{*}$ & $\mathrm{t}_{2}^{*}$ & $\mathrm{Tac}_{\mathrm{sd}}\left(\mathrm{t}_{1}, \mathrm{t}_{2}\right)^{*}$ & $\mathrm{~L}_{\mathrm{m}}{ }^{*}$ \\
\hline 0.9031 & 0.4924 & 3828.560 & 494.6113 \\
\hline
\end{tabular}

(3). When $\widetilde{\mathrm{C}_{1}}$, and $\tilde{\mathrm{p}}$ are Triangular fuzzy numbers then the solution is given bellow table:

Table 4: Output Result in fuzzy environment for case-3.

\begin{tabular}{|c|c|c|c|}
\hline $\mathrm{t}_{1}^{*}$ & $\mathrm{t}_{2}^{*}$ & $\operatorname{Tac}_{\mathrm{sd}}\left(\mathrm{t}_{1}, \mathrm{t}_{2}\right)^{*}$ & $\mathrm{~L}_{\mathrm{m}}{ }^{*}$ \\
\hline 0.9031 & 0.4924 & 3828.560 & 494.6113 \\
\hline
\end{tabular}

(4). When only $\widetilde{\mathrm{C}_{1}}$ is Triangular fuzzy numbers then the solution is given bellow table:

Table 5: Output Result in fuzzy environment for case-4.

\begin{tabular}{|c|c|c|c|}
\hline $\mathrm{t}_{1}^{*}$ & $\mathrm{t}_{2}^{*}$ & $\mathrm{Tac}_{\mathrm{sd}}\left(\mathrm{t}_{1}, \mathrm{t}_{2}\right)^{*}$ & $\mathrm{~L}_{\mathrm{m}}{ }^{*}$ \\
\hline 0.9168 & 0.5023 & 3816.822 & 484.7281 \\
\hline
\end{tabular}

(5). When none of $\widetilde{\mathrm{C}_{1}}, \widetilde{\mathrm{p}}, \widetilde{\mathrm{C}_{2}}$ and $\tilde{\mathrm{h}}$ is a Triangular fuzzy numbers then the solution is given bellow table:

Table 6: Output Result in fuzzy environment for case-5.

\begin{tabular}{|c|c|c|c|}
\hline $\mathrm{t}_{1}^{*}$ & $\mathrm{t}_{2}^{*}$ & $\operatorname{Tac}_{\mathrm{sd}}\left(\mathrm{t}_{1}, \mathrm{t}_{2}\right)^{*}$ & $\mathrm{~L}_{\mathrm{m}}{ }^{*}$ \\
\hline 0.9168 & 0.5023 & 3816.822 & 484.7281 \\
\hline
\end{tabular}

Comparison of Optimal Solutions is given in Table-7:

\begin{tabular}{|c|c|c|c|c|}
\hline Model & Optimal value of $\mathrm{t}_{1}$ & Optimal value of $\mathrm{t}_{2}$ & Optimal value of Tac $\left(\mathrm{t}_{1}, \mathrm{t}_{2}\right)$ & Optimal value of $\mathrm{L}_{\mathrm{m}}$ \\
\hline Crisp & 0.9168 & 0.5023 & 3816.822 & 484.7281 \\
\hline Fuzzy & 0.9191 & 0.5040 & 3814.925 & 503.4081 \\
\hline
\end{tabular}


INDEPENDENT JOURNAL OF MANAGEMENT \& PRODUCTION (IJM\&P)

http://Www.ijmp.jor.br

V. 10, n. 5, September-October 2019

ISSN: 2236-269X

DOI: 10.14807/ijmp.v10i5.897

\section{SENSITIVITY ANALYSIS}

We currently analyse to sensitivity analysis of the optimal solution of the model for change system parameters $C_{1}, k, C_{2}, m, f, h, p, d, I, r$ and $\beta$ by $-30 \%,-15 \%,+15 \%$, $+30 \%$ individually, keeping alternate parameters unaltered. The underlying information is taken from the above numerical illustration.

Table 8: Sensitivity Analysis

\begin{tabular}{|c|c|c|c|c|c|c|}
\hline Parameters & Changed Value & ${ }^{*} \mathrm{PCPV}$ & $t_{1}^{*}$ & $\mathrm{t}_{2}^{*}$ & $\operatorname{Tac}\left(t_{1}, t_{2}\right)^{*}$ & $\mathrm{~L}_{\mathrm{m}}{ }^{*}$ \\
\hline \multirow{5}{*}{$C_{1}=175$} & 122.5 & -30 & 0.8572 & 0.4795 & 3778.723 & 453.2304 \\
\hline & 148.75 & -15 & 0.8875 & 0.4911 & 3798.057 & 469.2162 \\
\hline & 175 & 00 & 0.9168 & 0.5023 & 3816.822 & 484.7281 \\
\hline & 201.25 & +15 & 0.9454 & 0.5132 & 3835.066 & 499.8058 \\
\hline & 227.5 & +30 & 0.9731 & 0.5238 & 3852.839 & 514.4838 \\
\hline \multirow{5}{*}{$k=1.4$} & 0.98 & -30 & 0.0000 & 0.7685 & 4233.764 & 0.0000 \\
\hline & 1.19 & -15 & 1.7367 & 0.1635 & 3268.603 & 434.3065 \\
\hline & 1.4 & 00 & 0.9168 & 0.5023 & 3816.822 & 484.7281 \\
\hline & 1.61 & +15 & 0.4117 & 0.6943 & 4121.946 & 332.3405 \\
\hline & 1.82 & +30 & 0.0184 & 0.7681 & 4233.477 & 19.0962 \\
\hline \multirow{5}{*}{$C_{2}=50$} & 35 & -30 & 0.8123 & 0.4622 & 3750.002 & 429.4780 \\
\hline & 42.5 & -15 & 0.8660 & 0.4828 & 3784.308 & 457.8487 \\
\hline & 50 & 00 & 0.9168 & 0.5023 & 3816.822 & 484.7281 \\
\hline & 57.5 & +15 & 0.9653 & 0.5208 & 3847.801 & 510.3290 \\
\hline & 65 & +30 & 1.0115 & 0.5385 & 3877.443 & 534.8182 \\
\hline \multirow{5}{*}{$m=2565$} & 1795.5 & -30 & 1.1219 & 0.5797 & 2762.855 & 414.5397 \\
\hline & 2080.25 & -15 & 1.0329 & 0.5462 & 3155.231 & 442.4768 \\
\hline & 2565 & 00 & 0.9168 & 0.5023 & 3816.822 & 484.7281 \\
\hline & 2949.75 & +15 & 0.8459 & 0.4753 & 4337.446 & 514.5294 \\
\hline & 3334.5 & +30 & 0.7875 & 0.4529 & 4854.907 & 541.6986 \\
\hline \multirow{5}{*}{$f=6$} & 4.2 & -30 & 0.9975 & 0.5325 & 2707.156 & 308.5458 \\
\hline & 5.1 & -15 & 0.9508 & 0.5150 & 3262.388 & 426.9689 \\
\hline & 6 & 00 & 0.9168 & 0.5023 & 3816.822 & 484.7281 \\
\hline & 6.9 & +15 & 0.8911 & 0.4926 & 4370.720 & 542.0407 \\
\hline & 7.8 & +30 & 0.8708 & 0.4850 & 4924.238 & 599.0377 \\
\hline \multirow{5}{*}{$h=1.2$} & 0.84 & -30 & 1.0638 & 0.6151 & 3709.147 & 562.4372 \\
\hline & 1.02 & -15 & 0.9820 & 0.5509 & 3765.363 & 519.1984 \\
\hline & 1.2 & 00 & 0.9168 & 0.5023 & 3816.822 & 484.7281 \\
\hline & 1.38 & +15 & 0.8633 & 0.4640 & 3864.551 & 456.4212 \\
\hline & 1.56 & +30 & 0.8183 & 0.4328 & 3909.253 & 432.6353 \\
\hline \multirow{5}{*}{$p=11.6$} & 8.12 & -30 & 0.7844 & 0.4104 & 3945.814 & 593.0900 \\
\hline & 9.86 & -15 & 0.8545 & 0.4581 & 3872.760 & 531.7821 \\
\hline & 11.6 & 00 & 0.9168 & 0.5023 & 3816.822 & 484.7281 \\
\hline & 13.35 & +15 & 0.9743 & 0.5447 & 3771.299 & 446.3356 \\
\hline & 15.08 & +30 & 1.0244 & 0.5829 & 3735.587 & 416.1339 \\
\hline \multirow{7}{*}{$d=1.3$} & 0.91 & -30 & 0.1356 & 0.7619 & 3325.834 & 71.7164 \\
\hline & 1.105 & -15 & 0.6054 & 0.6614 & 3621.825 & 320.0902 \\
\hline & 1.3 & 00 & 0.9168 & 0.5023 & 3816.822 & 484.7281 \\
\hline & 1.425 & +15 & 1.1212 & 0.3035 & 3944.362 & 592.7702 \\
\hline & 1.69 & +30 & 1.2193 & 0.0642 & 4005.503 & 644.6292 \\
\hline & 0.105 & -30 & 0.9551 & 0.4762 & 2870.387 & 504.9487 \\
\hline & 0.1275 & -15 & 0.9363 & 0.4894 & 3343.782 & 495.0048 \\
\hline
\end{tabular}


INDEPENDENT JOURNAL OF MANAGEMENT \& PRODUCTION (IJM\&P)

http://Www.ijmp.jor.br

V. 10, n. 5, September-October 2019

ISSN: 2236-269X

DOI: 10.14807/ijmp.v10i5.897

\begin{tabular}{|c|c|c|c|c|c|c|}
\hline \multirow{3}{*}{$l=0.15$} & 0.15 & 00 & 0.9168 & 0.5023 & 3816.822 & 484.7281 \\
\cline { 2 - 6 } & 0.1725 & +15 & 0.8968 & 0.5150 & 4289.504 & 474.1139 \\
\cline { 2 - 6 } & 0.195 & +30 & 0.8760 & 0.5274 & 4761.822 & 463.1572 \\
\hline \multirow{4}{*}{$r=2$} & 1.4 & -30 & 0.9161 & 0.5024 & 3817.035 & 484.9041 \\
\cline { 2 - 6 } & 1.7 & -15 & 0.9165 & 0.5024 & 3816.929 & 484.8161 \\
\cline { 2 - 6 } & 2 & 00 & 0.9168 & 0.5023 & 3816.822 & 484.7281 \\
\cline { 2 - 6 } & 2.3 & +15 & 0.9172 & 0.5022 & 3816.716 & 484.600 \\
\hline \multirow{4}{*}{$\beta=0.01$} & 2.6 & +30 & 0.9176 & 0.5022 & 3816.609 & 484.5518 \\
\cline { 2 - 6 } & 0.0070 & -30 & 0.9172 & 0.5048 & 3816.068 & 484.8918 \\
\cline { 2 - 6 } & 0.0085 & -15 & 0.9170 & 0.5038 & 3816.446 & 484.8086 \\
\cline { 2 - 6 } & 0.0100 & 00 & 0.9168 & 0.5023 & 3816.822 & 484.7281 \\
\cline { 2 - 6 } & 0.0115 & +15 & 0.9167 & 0.5011 & 3817.197 & 484.6501 \\
\hline & 0.0130 & +30 & 0.9153 & 0.4865 & 3821.674 & 483.8837 \\
\hline
\end{tabular}

${ }^{*} \mathrm{PCPV}=$ Percentage Change in Parameter Values.

\section{OBSERVATIONS:}

From the above table we can close the accompanying:

(1). From the above table, for increasing of $C_{1}$, the optimal value of $t_{1}^{*}$ and $t_{2}^{*}$ increase slowly. By this effect, the total average cost $\operatorname{Tac}\left(t_{1}, t_{2}\right)^{*}$ and the highest inventory level $\mathrm{L}_{\mathrm{m}}{ }^{*}$ increase slowly. Bellow the graph to illustrate these results:

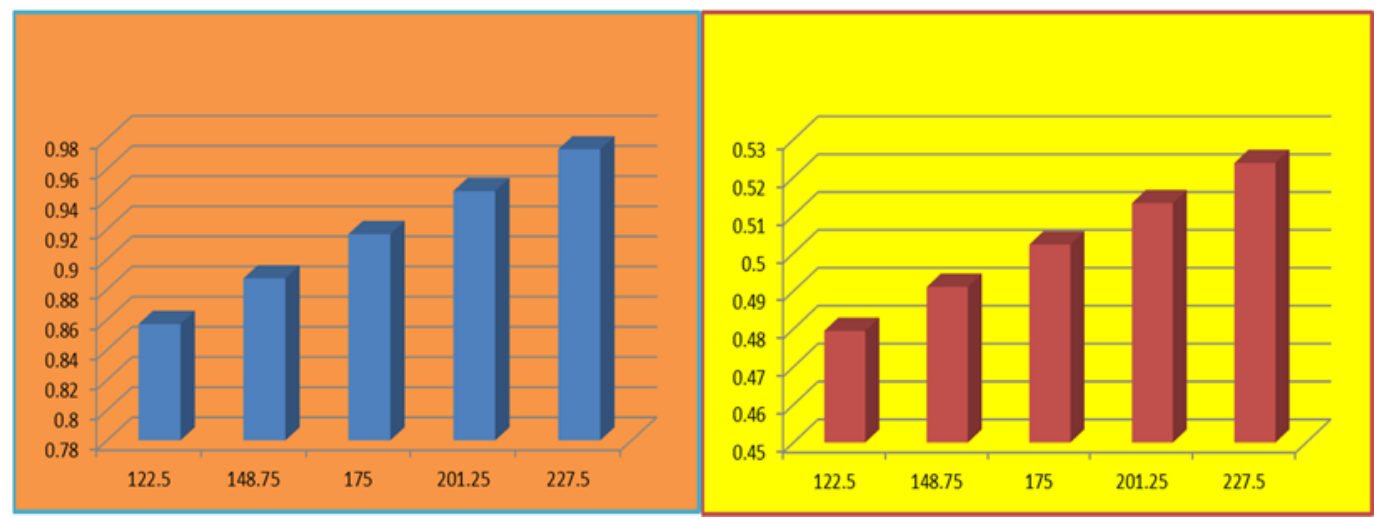

Graph for $C_{1} v s t^{*}$ in case-1

Graph for $C_{1}$ vs $t_{2}^{*}$ in case-1

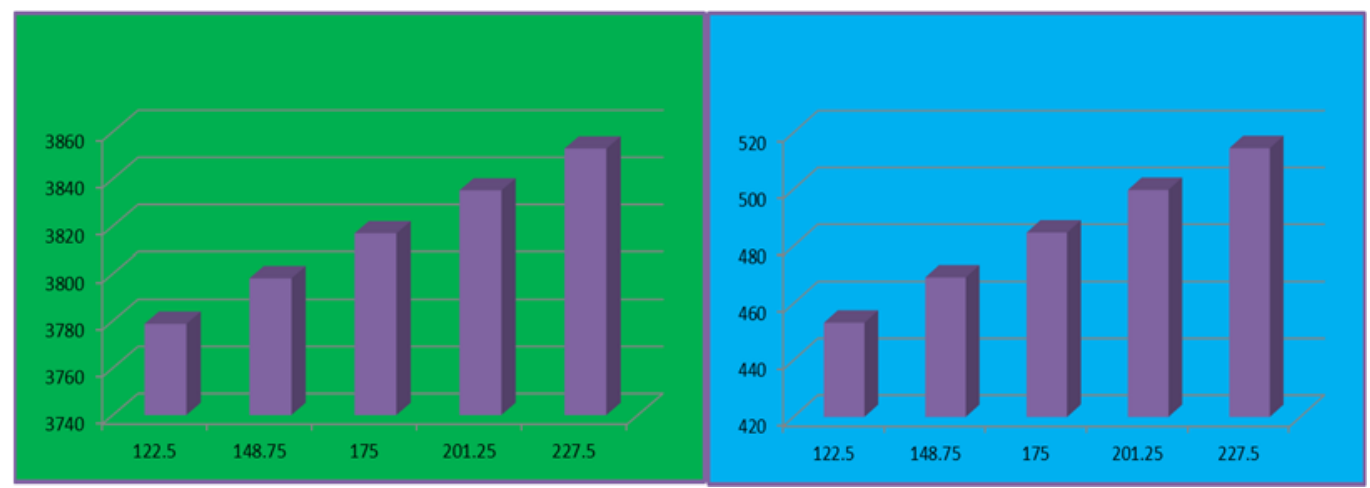

Graph for $C_{1} v s \operatorname{Tac}\left(\mathrm{t}_{1}, \mathrm{t}_{2}\right)^{*}$ in case-1 Graph for $C_{1} v s \mathrm{~L}_{\mathrm{m}}{ }^{*}$ in case-1 
INDEPENDENT JOURNAL OF MANAGEMENT \& PRODUCTION (IJM\&P)

http://www.ijmp.jor.br

V. 10, n. 5, September-October 2019

ISSN: 2236-269X

DOI: 10.14807/ijmp.v10i5.897

(2). From the above table, when $k<1$ i.e. 0.98 , the optimal value of $\mathrm{t}_{1}^{*}$ and the highest inventory level $\mathrm{L}_{\mathrm{m}}{ }^{*}$ become zero where the optimal value of $\mathrm{t}_{2}^{*}$ increase. With this effect the total average cost $\operatorname{Tac}\left(t_{1}, t_{2}\right)^{*}$ increase. Apart from this, for increasing of $k$, the optimal value of $t_{1}^{*}$ decrease and the optimal value of $t_{2}^{*}$ increase rapidly. By this effect, the total average cost $\operatorname{Tac}\left(\mathrm{t}_{1}, \mathrm{t}_{2}\right)^{*}$ increase and the highest inventory level $\mathrm{L}_{\mathrm{m}}{ }^{*}$ decrease rapidly. Bellow the graph to illustrate these results:

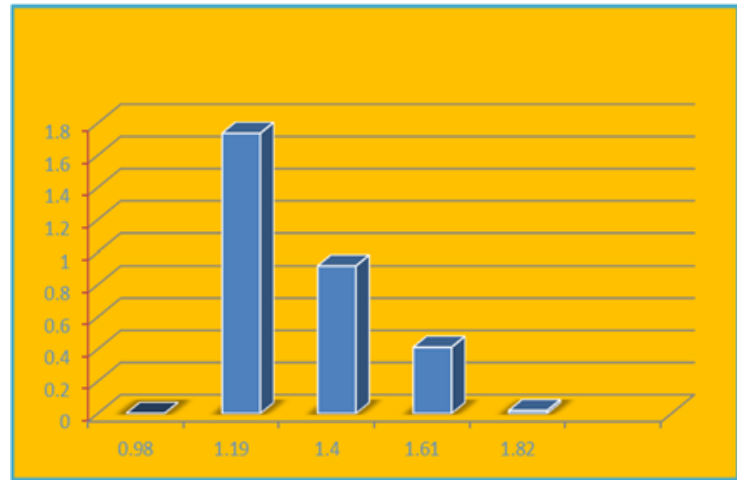

Graph for $k v s t^{*}{ }_{1}$ in case-2

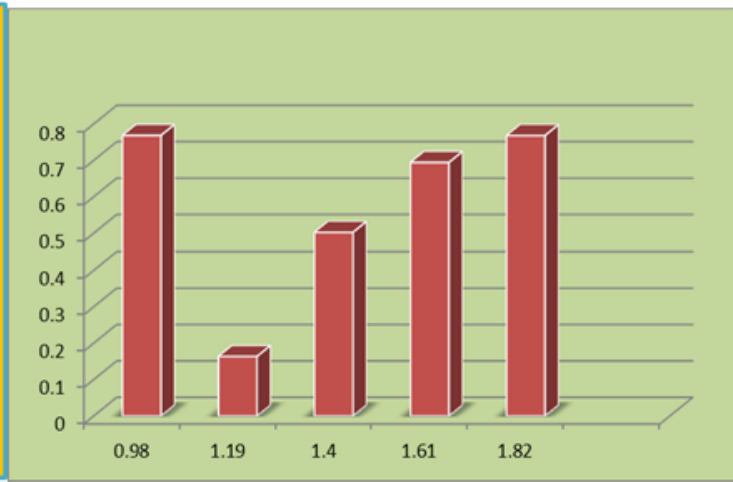

Graph for $k v s t^{*}{ }_{2}$ in case-2

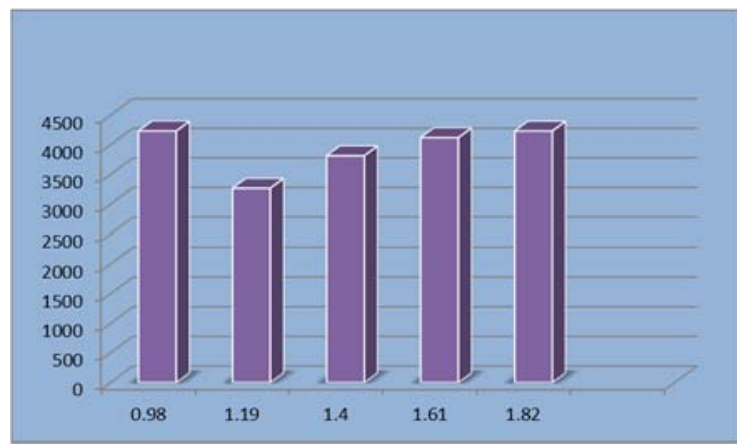

Graph for $k$ vs Tac $\left(\mathrm{t}_{1}, \mathrm{t}_{2}\right)^{*}$ in case-2

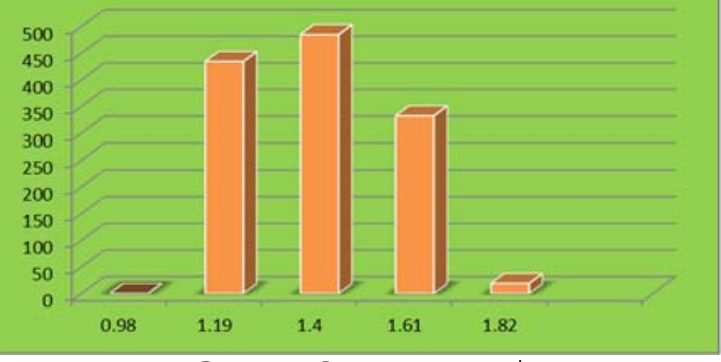

Graph for $k$ vs $\mathrm{L}_{\mathrm{m}}{ }^{*}$ in case-2

(3). From the above table, for increasing of $\mathrm{C}_{2}$, the optimal value of $t_{1}^{*}$ and $t_{2}^{*}$ increase slowly. By this effect, the total average cost $\operatorname{Tac}\left(t_{1}, t_{2}\right)^{*}$ increase slowly and the highest inventory level $\mathrm{L}_{\mathrm{m}}{ }^{*}$ increase rapidly. Bellow the graph to illustrate these results:

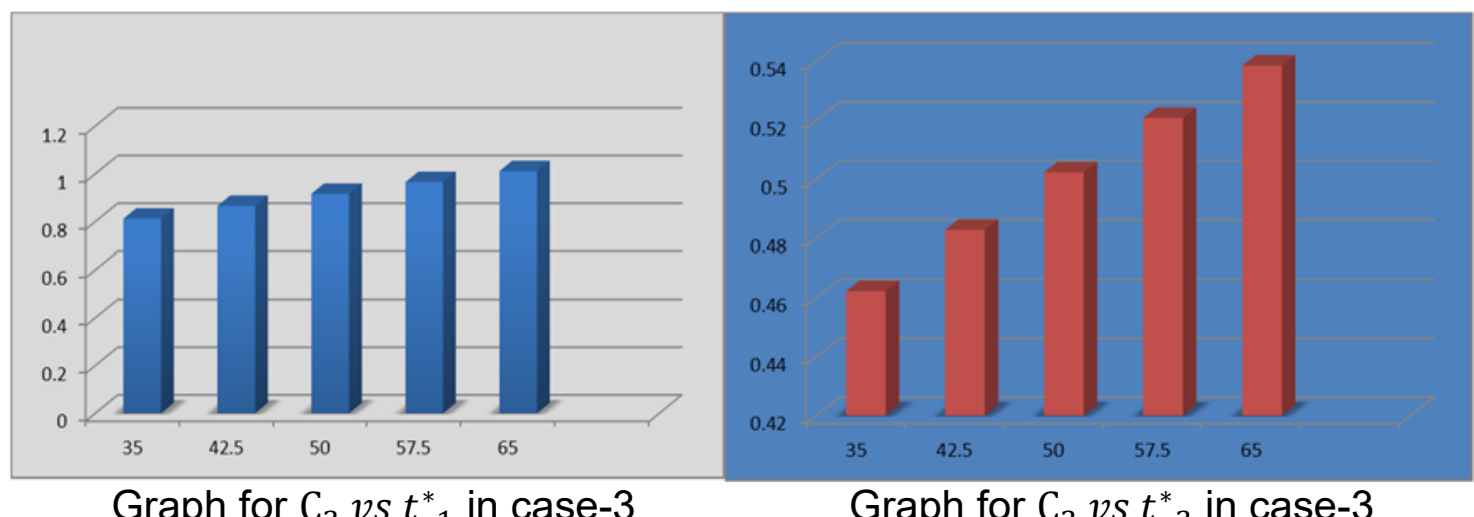

Graph for $\mathrm{C}_{2}$ vs $t^{*}{ }_{1}$ in case-3

Graph for $\mathrm{C}_{2}$ vs $t^{*}{ }_{2}$ in case-3 
INDEPENDENT JOURNAL OF MANAGEMENT \& PRODUCTION (IJM\&P)

http://www.ijmp.jor.br

V. 10, n. 5, September-October 2019

ISSN: 2236-269X

DOI: 10.14807/ijmp.v10i5.897

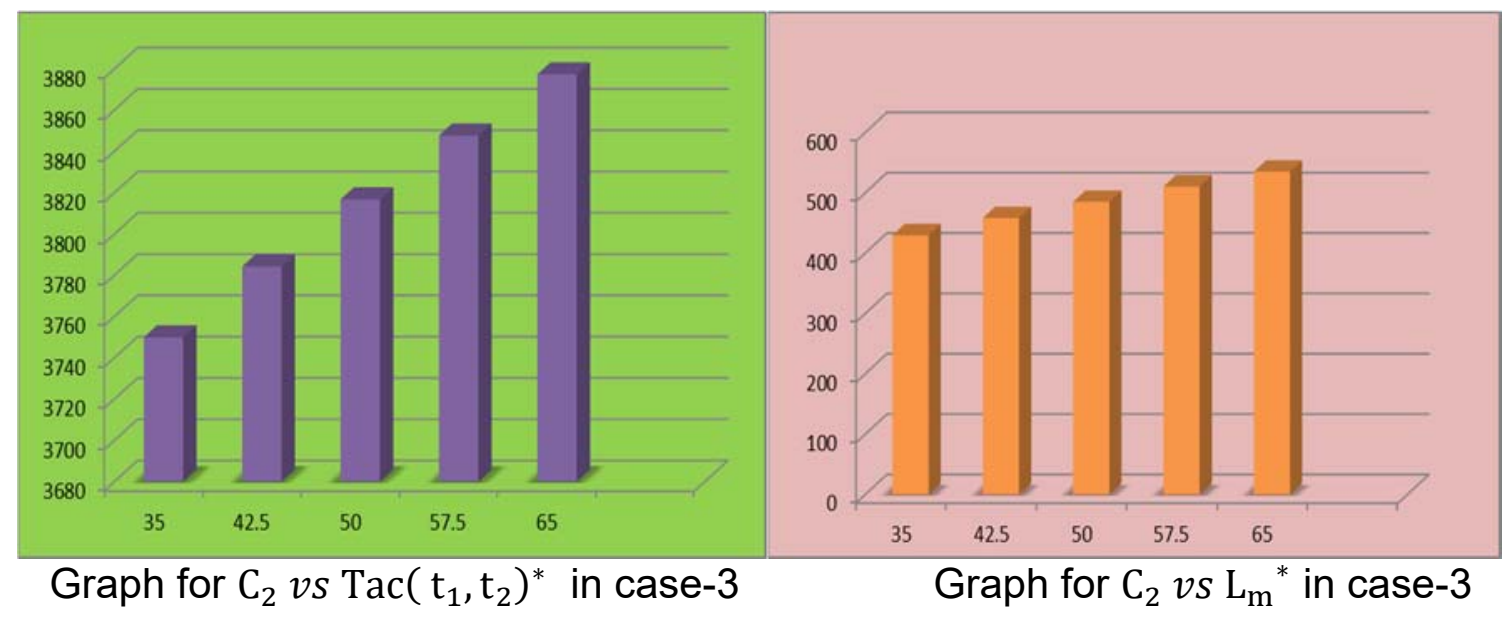

(4). From the above table, for increasing of $m$, the optimal value of $t_{1}^{*}$ and $t_{2}^{*}$ decrease slowly. By this effect, the total average $\operatorname{cost} \operatorname{Tac}\left(t_{1}, t_{2}\right)^{*}$ and the highest inventory level $\mathrm{L}_{\mathrm{m}}{ }^{*}$ increase rapidly. Bellow the graph to illustrate these results:

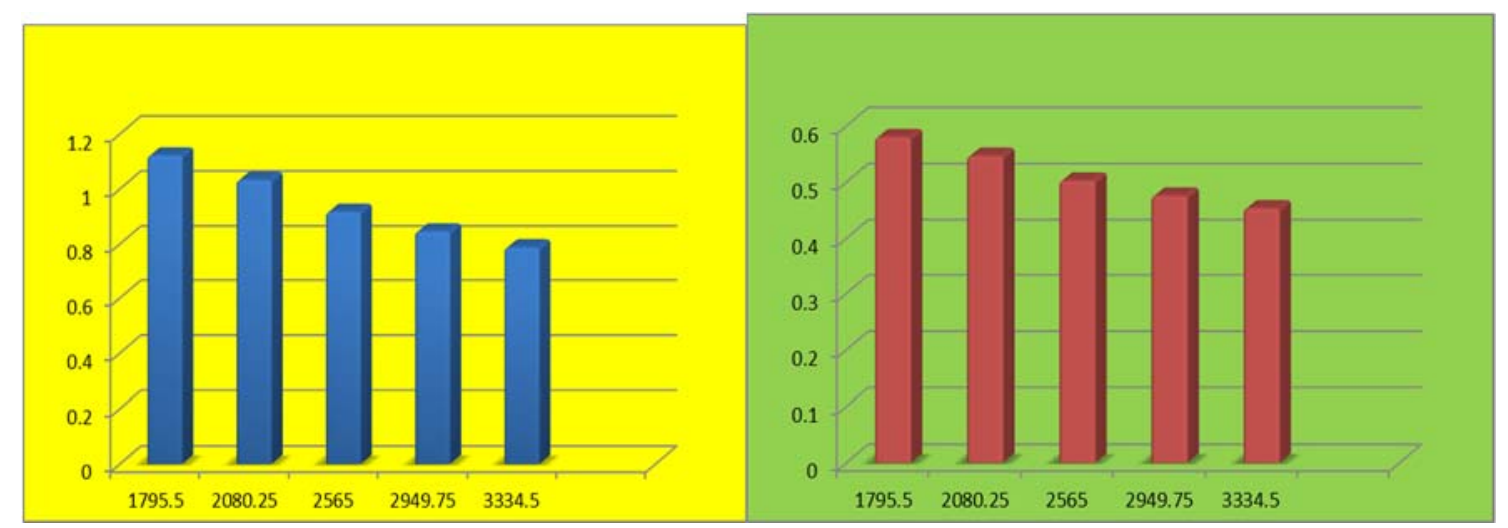

Graph for $\mathrm{m} v s t^{*}{ }_{1}$ in case-4

Graph for $\mathrm{m} v s t^{*}{ }_{2}$ in case-4

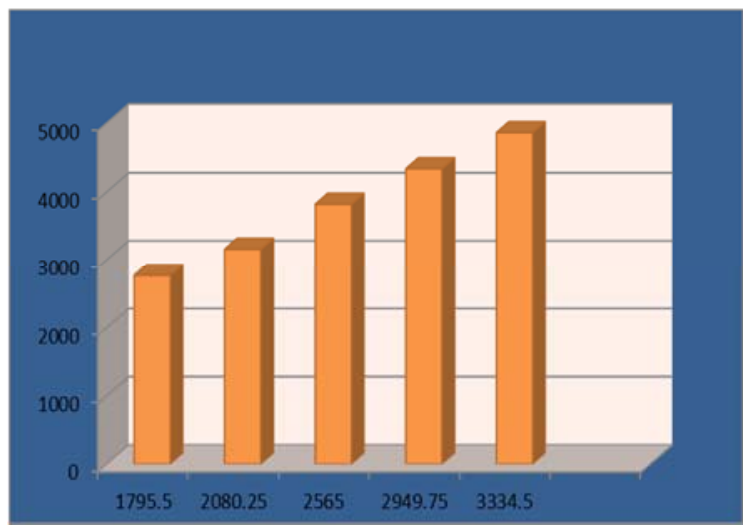

Graph for $\mathrm{m} v s \operatorname{Tac}\left(\mathrm{t}_{1}, \mathrm{t}_{2}\right)^{*}$ in case-4

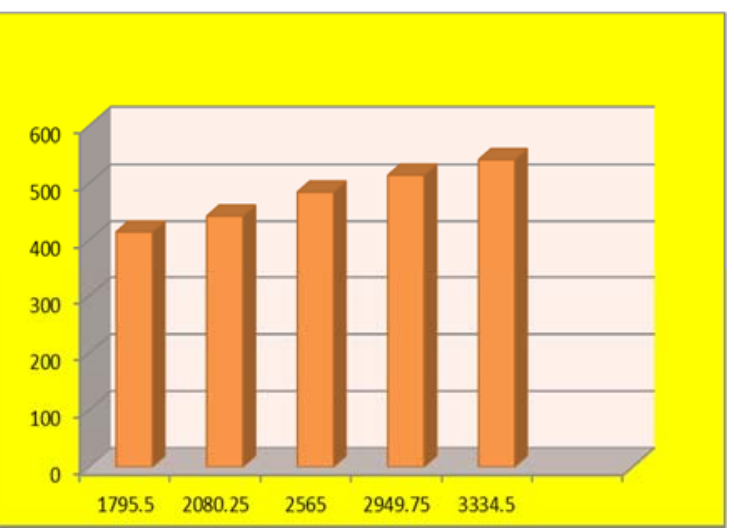

Graph for $\mathrm{m} v s \mathrm{~L}_{\mathrm{m}}{ }^{*}$ in case-4

(5). From the above table, for increasing of $f$, the optimal value of $t_{1}^{*}$ and $t_{2}^{*}$ decrease slowly. By this effect, the total average $\operatorname{cost} \operatorname{Tac}\left(t_{1}, t_{2}\right)^{*}$ and the highest inventory level $\mathrm{L}_{\mathrm{m}}{ }^{*}$ increase rapidly. Bellow the graph to illustrate these results: 
INDEPENDENT JOURNAL OF MANAGEMENT \& PRODUCTION (IJM\&P)

http://Www.ijmp.jor.br

V. 10, n. 5, September-October 2019

ISSN: 2236-269X

DOI: 10.14807/ijmp.v10i5.897

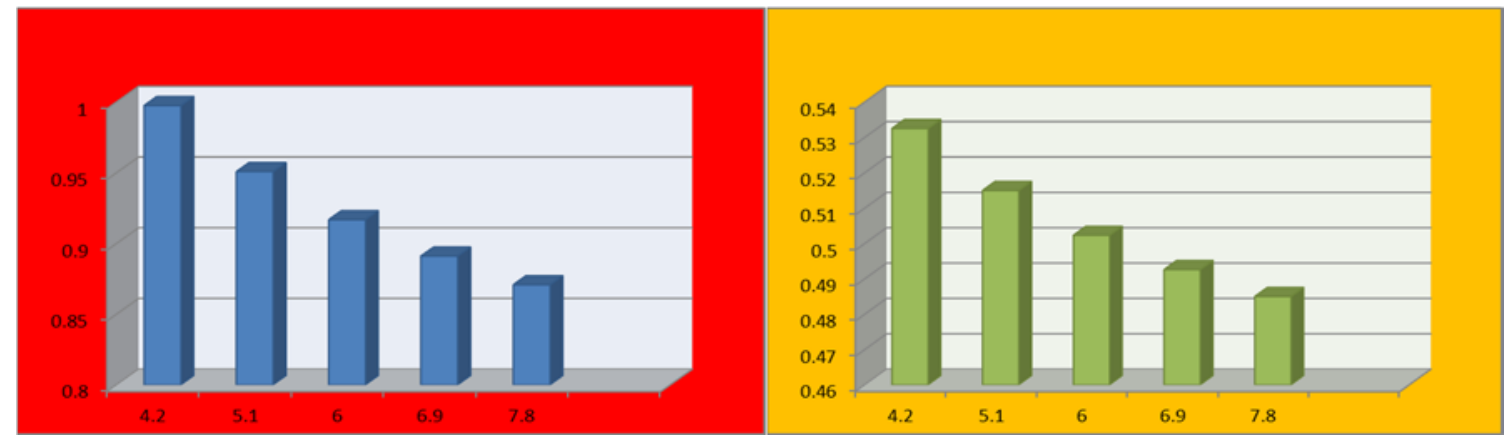

Graph for $\mathrm{f} v s t^{*}$ in case-5

Graph for $\mathrm{f} v s t_{2}^{*}$ in case-5

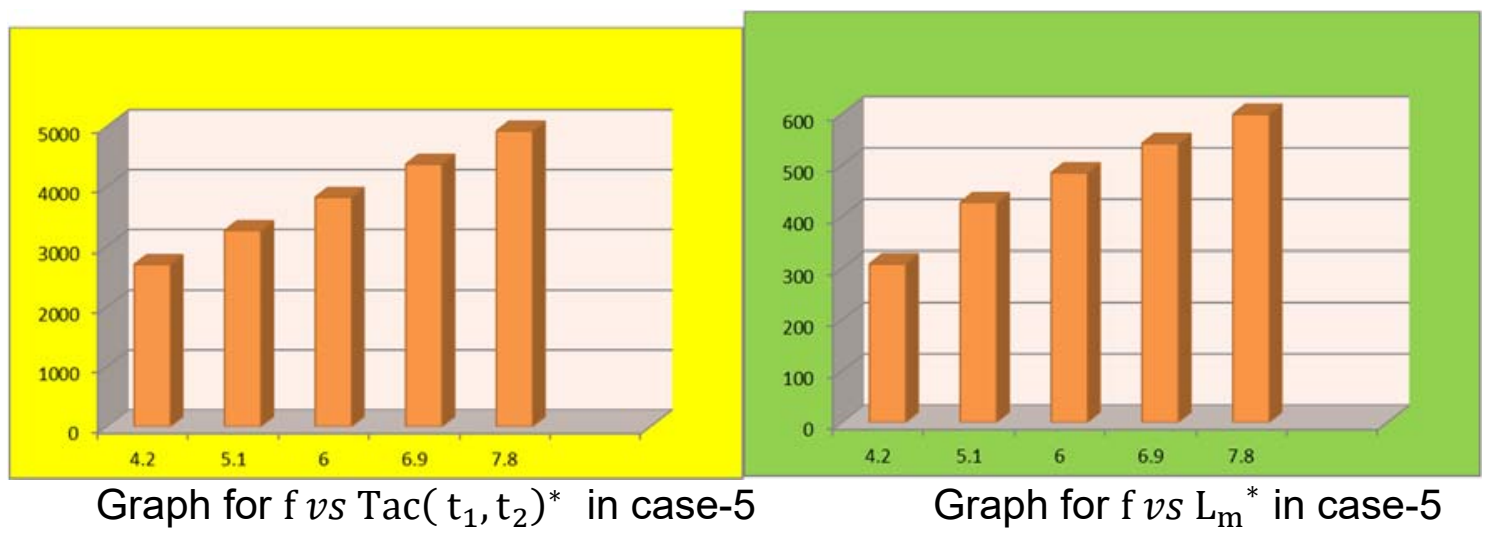

(6) From the above table, for increasing of $h$, the optimal value of $t_{1}^{*}$ and $t_{2}^{*}$ decrease slowly. By this effect, the total average cost $\operatorname{Tac}\left(t_{1}, t_{2}\right)^{*}$ increase and the highest inventory level $\mathrm{L}_{\mathrm{m}}{ }^{*}$ decrease slowly. Bellow the graph to illustrate these results:

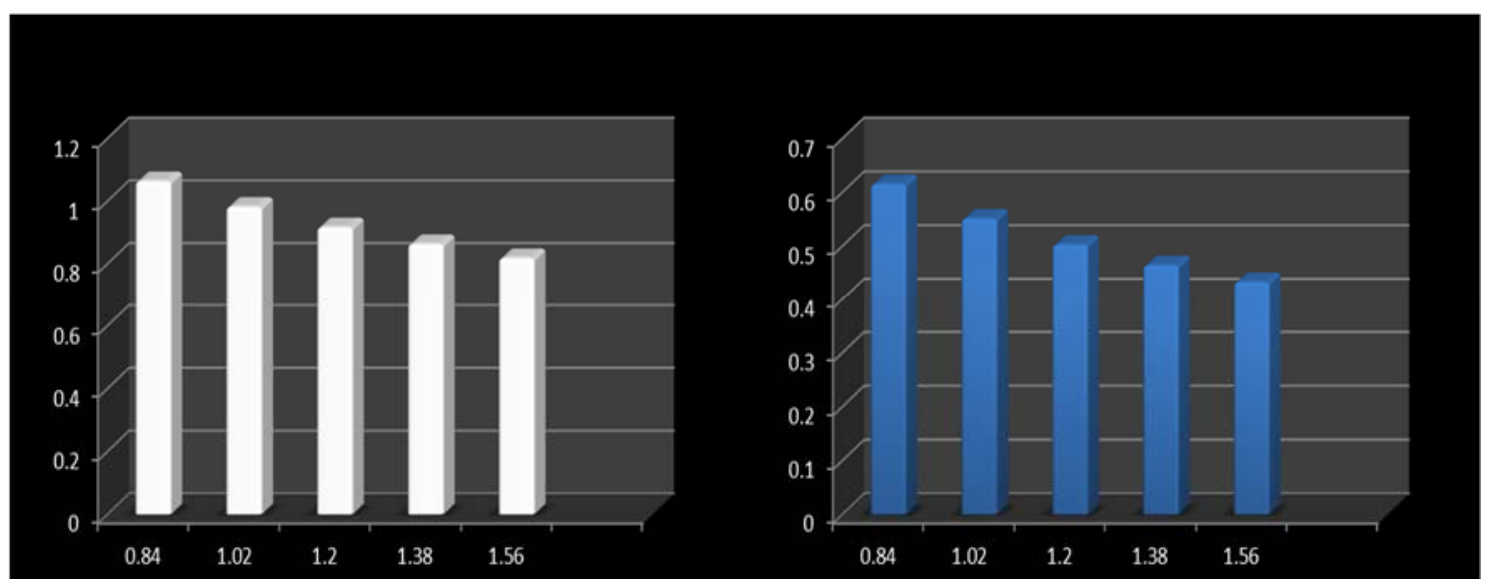

Graph for $\mathrm{h} v s t^{*}$ in case-6

Graph for h $v s t^{*}{ }_{2}$ in case-6 
INDEPENDENT JOURNAL OF MANAGEMENT \& PRODUCTION (IJM\&P)

http://Www.ijmp.jor.br

V. 10, n. 5, September-October 2019

ISSN: 2236-269X

DOI: 10.14807/ijmp.v10i5.897

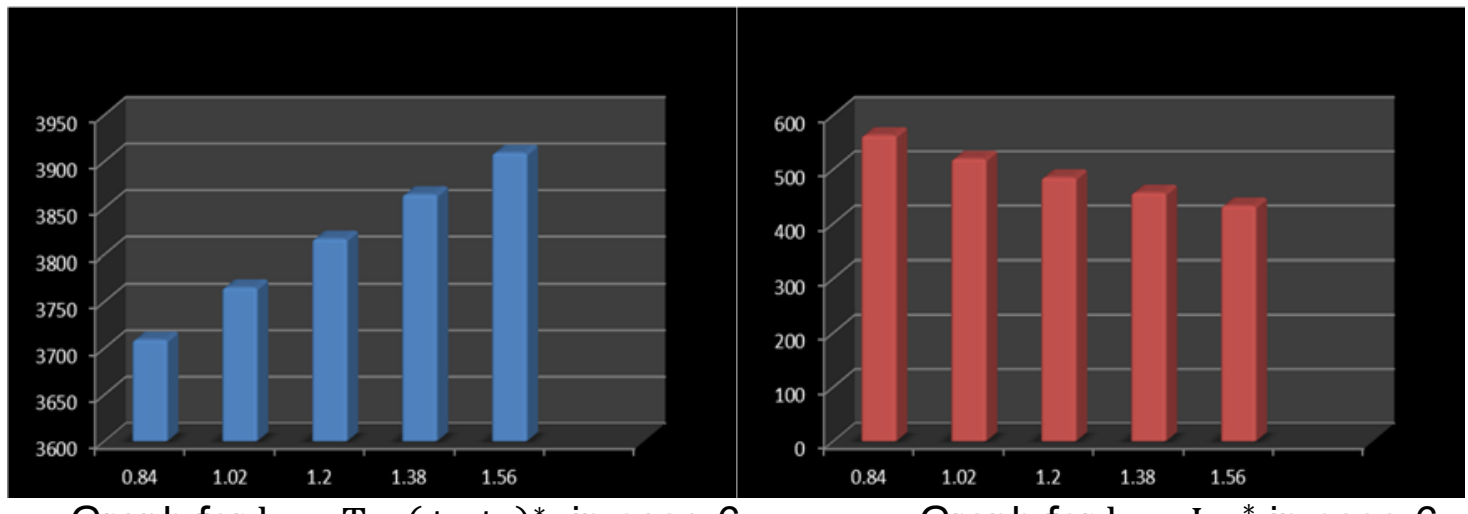

Graph for $h v s \operatorname{Tac}\left(\mathrm{t}_{1}, \mathrm{t}_{2}\right)^{*}$ in case-6

Graph for h $v s \mathrm{~L}_{\mathrm{m}}{ }^{*}$ in case-6

(7). From the above table, for increasing of $p$, the optimal value of $t_{1}^{*}$ increase rapidly and the optimal value of $t_{2}^{*}$ increase slowly. By this effect, the total average cost $\operatorname{Tac}\left(t_{1}, t_{2}\right)^{*}$ decrease slowly and the highest inventory level $\mathrm{L}_{\mathrm{m}}{ }^{*}$ decrease rapidly. Bellow the graph to illustrate these results:
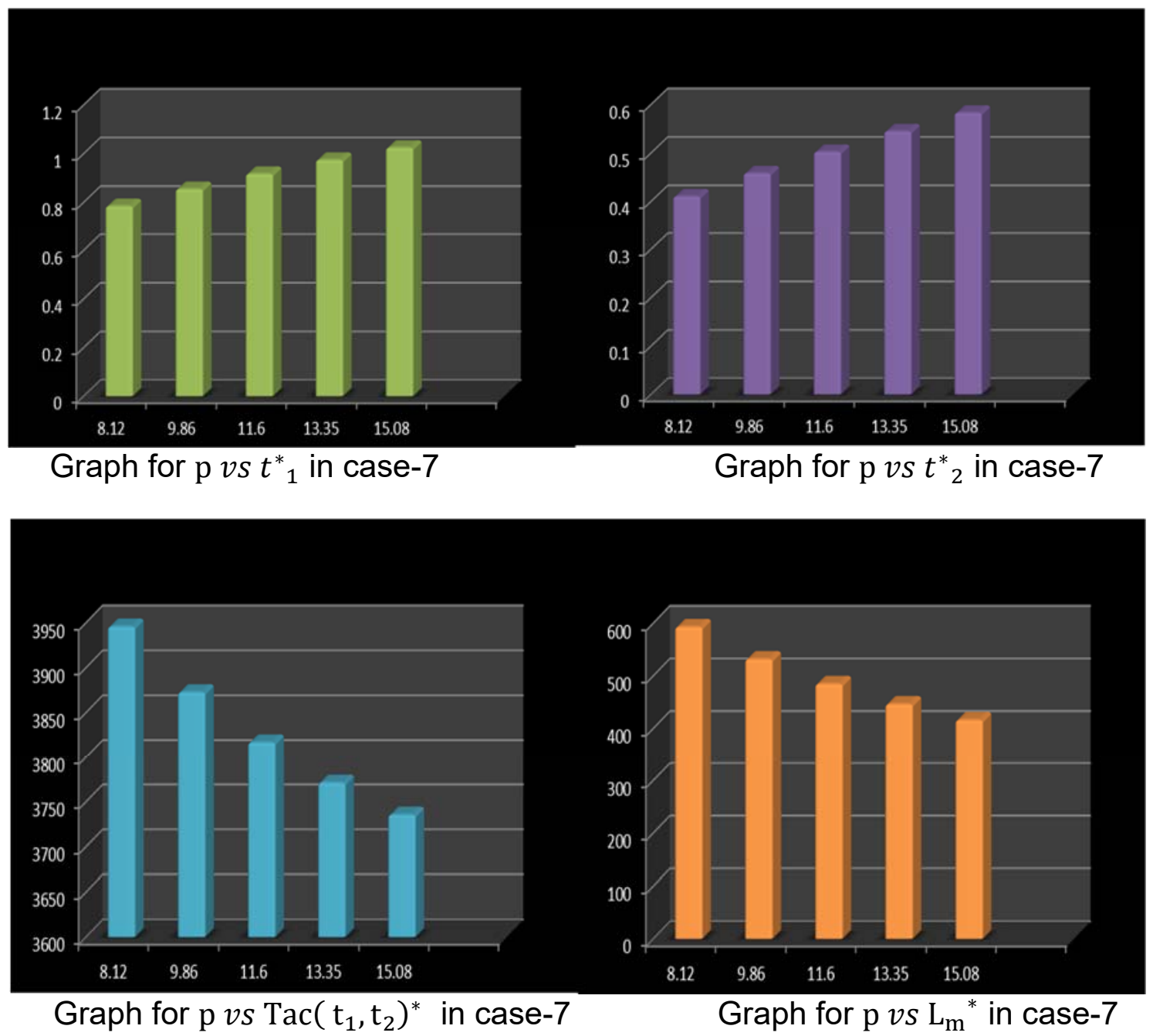
INDEPENDENT JOURNAL OF MANAGEMENT \& PRODUCTION (IJM\&P)

http://www.ijmp.jor.br

V. 10, n. 5, September-October 2019

ISSN: 2236-269X

DOI: $10.14807 /$ ijmp.v10i5.897

(8) From the above table, for increasing of $d$, the optimal value of $t_{1}^{*}$ increase and the optimal value of $t_{2}^{*}$ decrease rapidly. By this effect, the total average $\operatorname{cost} \operatorname{Tac}\left(t_{1}, t_{2}\right)^{*}$ increase slowly and the highest inventory level $\mathrm{L}_{\mathrm{m}}{ }^{*}$ increase rapidly. Bellow the graph to illustrate these results:

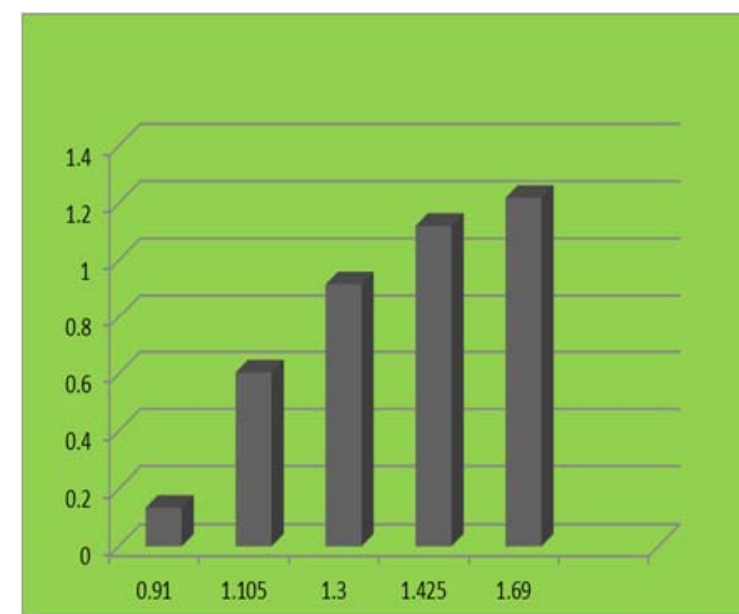

Graph for $\mathrm{d} v s t^{*}{ }_{1}$ in case-8

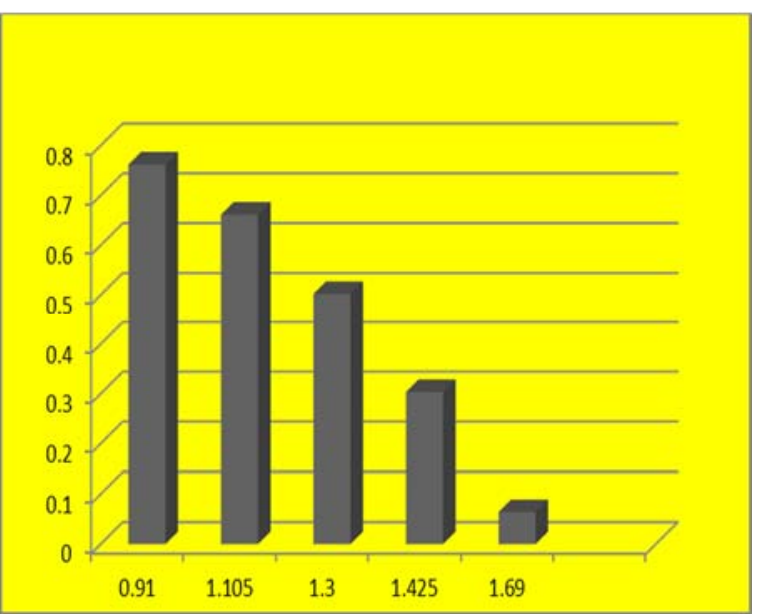

Graph for $\mathrm{d} v s t_{2}^{*}$ in case-8

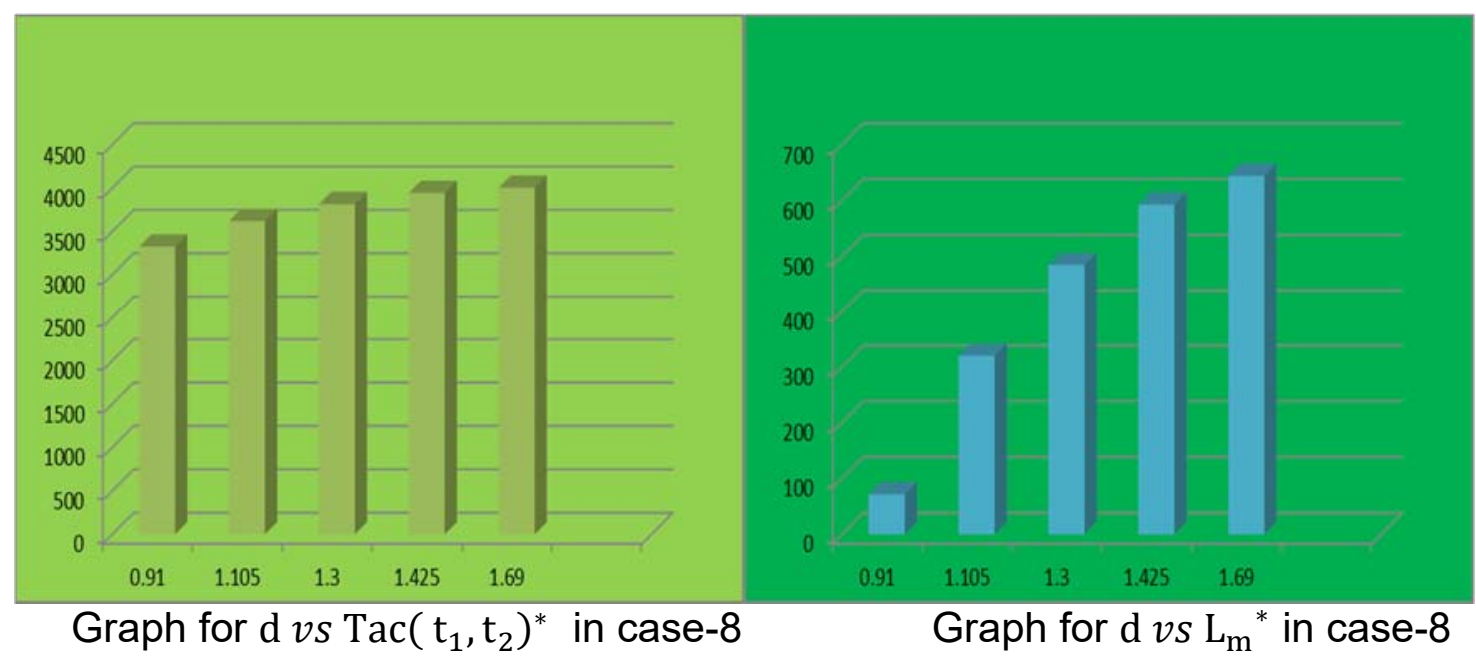

(9) From the above table, for increasing of $I$, the optimal value of $t_{1}^{*}$ decrease and $t_{2}^{*}$ increase slowly. By this effect, the total average cost $\operatorname{Tac}\left(t_{1}, t_{2}\right)^{*}$ increase rapidly and the highest inventory level $\mathrm{L}_{\mathrm{m}}{ }^{*}$ decrease slowly. Bellow the graph to illustrate these results: 
INDEPENDENT JOURNAL OF MANAGEMENT \& PRODUCTION (IJM\&P)

http://Www.ijmp.jor.br

V. 10, n. 5, September-October 2019

ISSN: 2236-269X

DOI: 10.14807/ijmp.v10i5.897

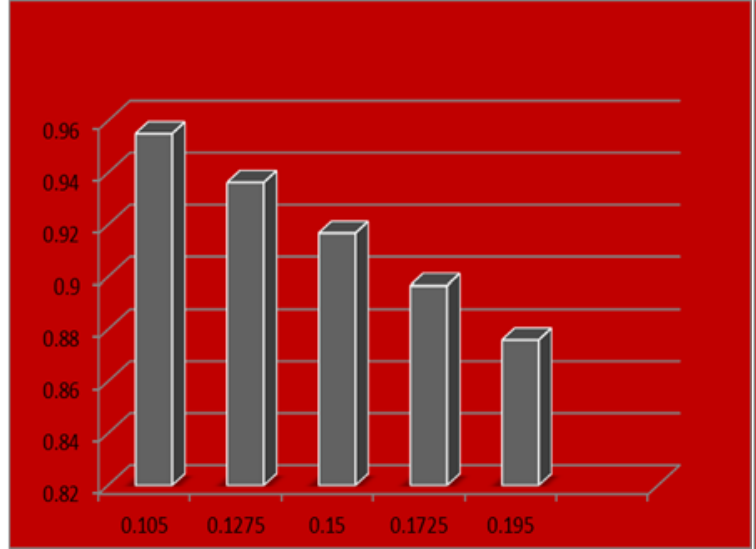

Graph for $1 v s t^{*}$ in case-9

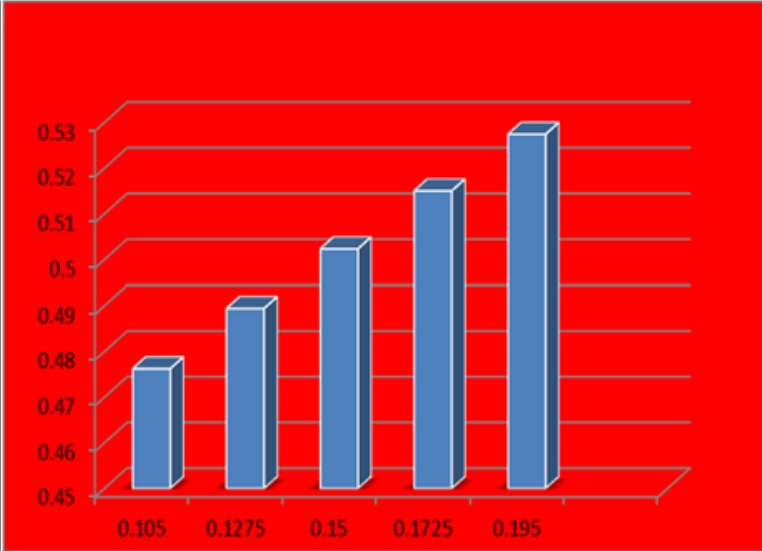

Graph for 1 vs $t_{2}^{*}$ in case-9

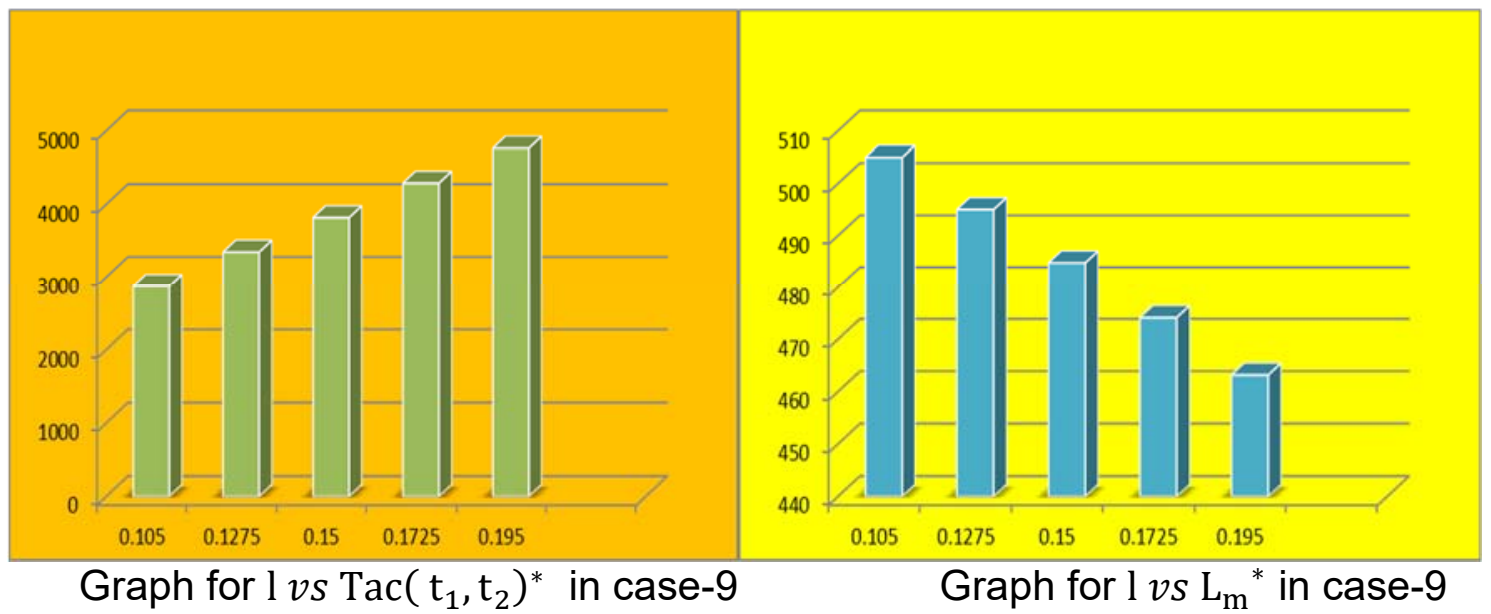

(10) From the above table, for increasing of $r$, the optimal value of $t_{1}^{*}$ increase and the optimal value of $\mathrm{t}_{2}^{*}$ decrease slightly. By this effect, the increment of the total average cost $\operatorname{Tac}\left(t_{1}, t_{2}\right)^{*}$ and the decrement of the highest inventory level $L_{m}{ }^{*}$ is negligible. Bellow the graph to illustrate these results:

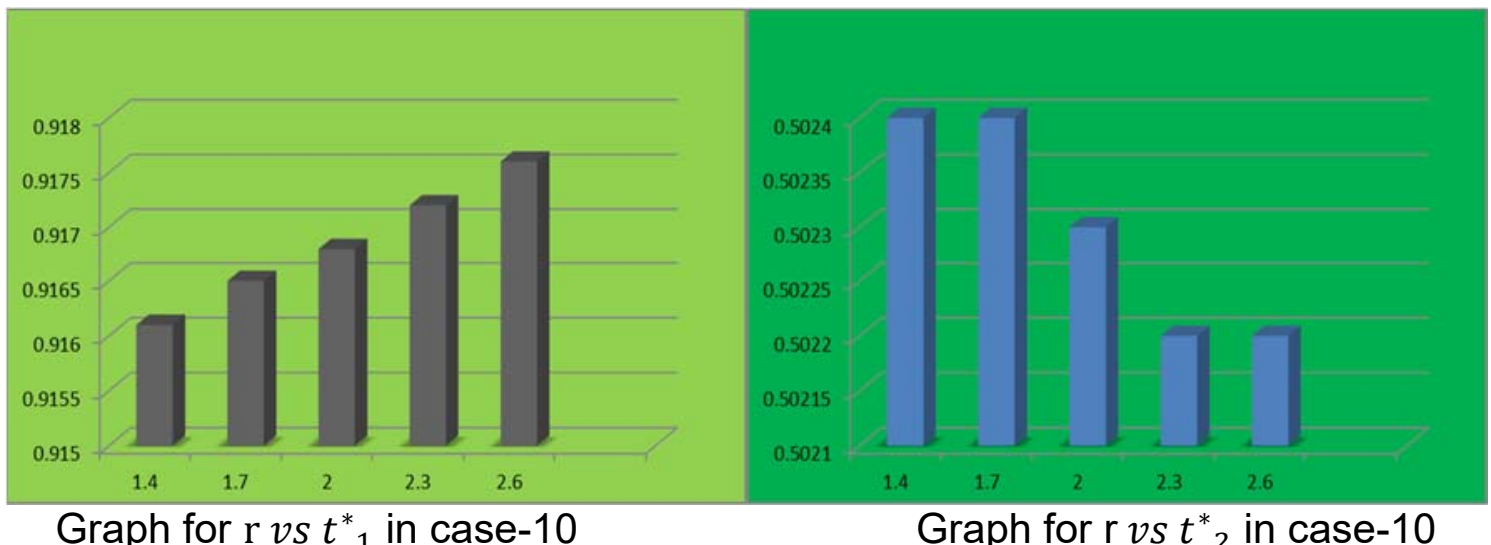




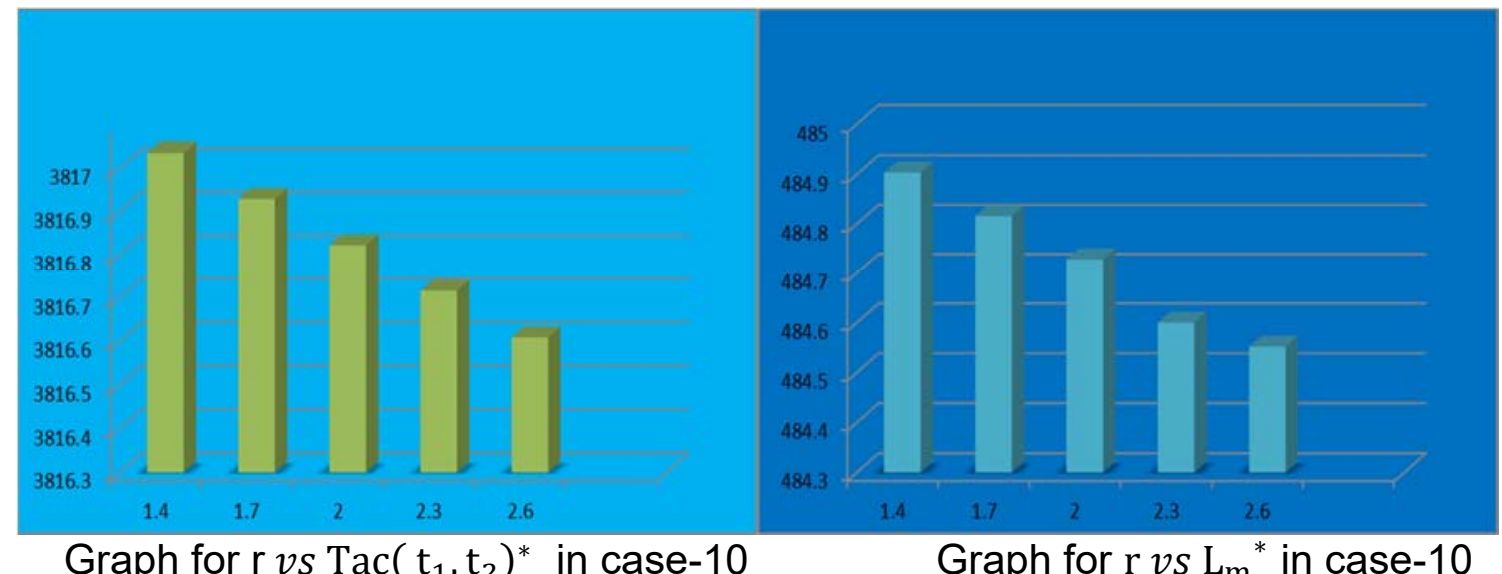

(11) From the above table, for increasing of $\beta$, the decrement of the optimal value of $t_{1}^{*}$ and $t_{2}^{*}$ is negligible. By this effect, the increment of the total average $\operatorname{cost} \operatorname{Tac}\left(t_{1}, t_{2}\right)^{*}$ is very slow and the decrement of the highest inventory level $\mathrm{L}_{\mathrm{m}}{ }^{*}$ is negligible. Bellow the graph to illustrate these results:

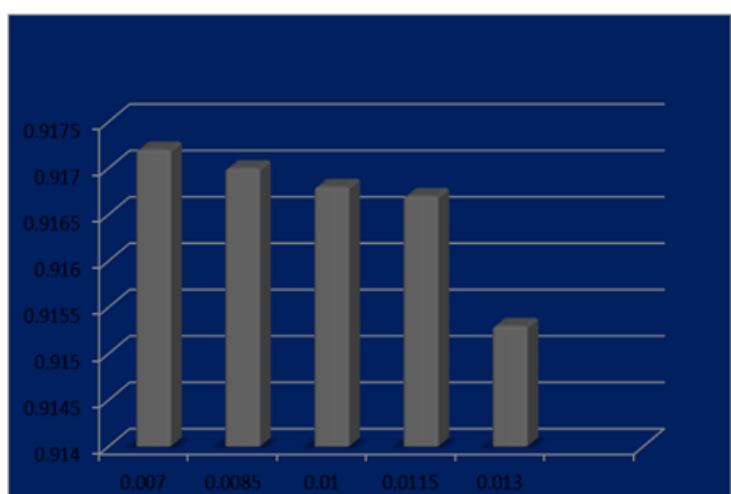

Graph for $\beta v s t^{*}$ in case-11

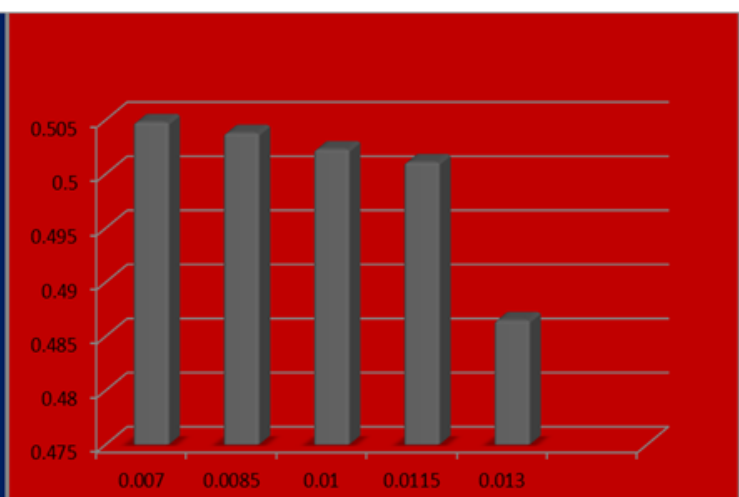

Graph for $\beta v s t_{2}^{*}$ in case-11

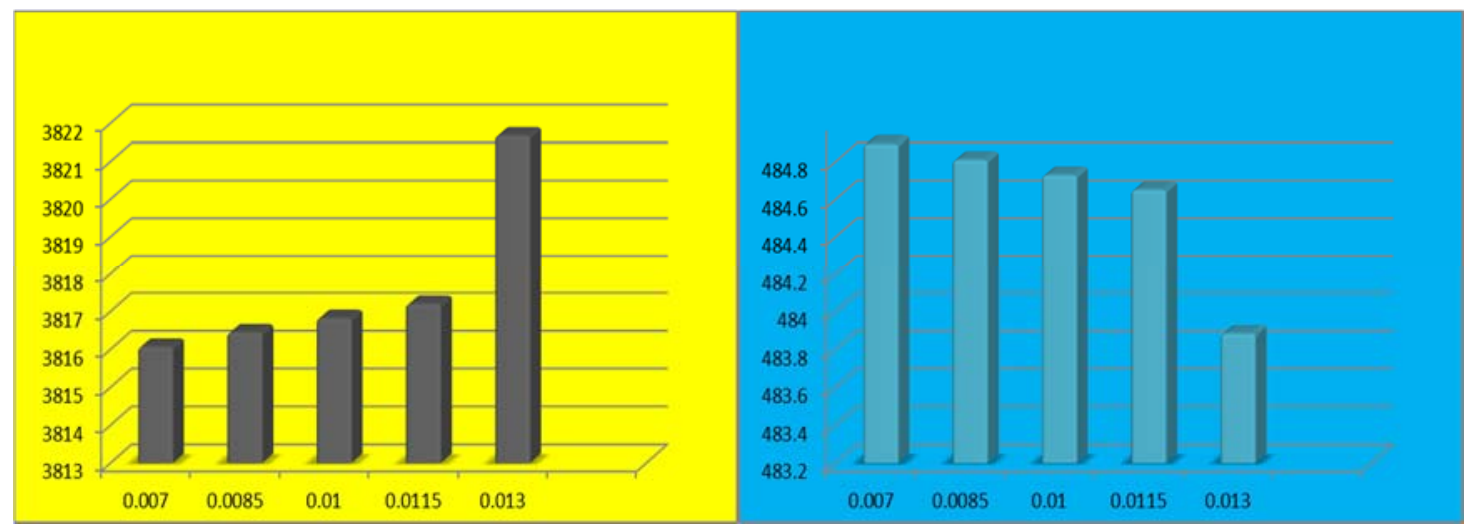

Graph for $\beta$ vs $\operatorname{Tac}\left(\mathrm{t}_{1}, \mathrm{t}_{2}\right)^{*}$ in case-11

Graph for $\beta$ vs $\mathrm{L}_{\mathrm{m}}{ }^{*}$ in case-11

\section{CONCLUSIONS}

In this article, we proposed a genuine E. P. Q. Inventory Model and gave solution along affectability examination approach. From the Table-9, it is indicates when deterioration, production cost, holding cost is lesser, average cost function of 
INDEPENDENT JOURNAL OF MANAGEMENT \& PRODUCTION (IJM\&P)

http://www.ijmp.jor.br

V. 10, n. 5, September-October 2019

ISSN: 2236-269X

DOI: 10.14807/ijmp.v10i5.897

the system decreases. Whereas it also observed that lesser population lesser demand and lesser selling price greater demand. Here, a crisp model is produced then it changed to fuzzy model taking triangular fuzzy number and illuminated by Signed Distance Method. Decision maker may get the ideal outcomes as per his desire utilizing the result of this model. In future, the other sort of membership functions, for example, Parabolic Fuzzy Number (pFN), Generalised Fuzzy Numbers, Piecewise Linear Hyperbolic Fuzzy Number, Parabolic level Fuzzy Number (PfFN), Pentagonal Fuzzy Number and so forth can be considered to build the membership function and afterward that model can be effectively solved by Werner's Approach, Nearest Interval Approximation, Geometric Programming (GP) strategy, Nearest Symmetric Triangular Defuzzification (NSTD) technique, and so forth.

\section{LIMITATIONS OF THE STUDY}

This proposed model of the inventory system there are a few constraints, which are as per the following:

1. The inventory system includes just a single thing and one stocking point.

2. The proposed model is restricted here on the grounds that shortages are not permitted.

3. This stock model diminishes the business chance up, as it were, yet this investigation does not ensure the end of business chance.

\section{FUTURE SCOPE}

In future, researchers can extend this model by taking allowable shortages, two warehouse, stock dependent demand, permissible delay in payment, stochastic demand and inflation. In furthere, the other sort of membership functions, for example, Parabolic Fuzzy Number (pFN), Generalised Fuzzy Numbers, Piecewise Linear Hyperbolic Fuzzy Number, Parabolic level Fuzzy Number (PfFN), Pentagonal Fuzzy Number and so forth can be considered to build the membership function and afterward that model can be effectively solved by Werner's Approach, Nearest Interval Approximation, Geometric Programming (GP) strategy, Nearest Symmetric Triangular Defuzzification (NSTD) technique, and so forth.

\section{ACKNOWLEDGEMENT}


INDEPENDENT JOURNAL OF MANAGEMENT \& PRODUCTION (IJM\&P)

http://Www.ijmp.jor.br

V. 10, n. 5, September-October 2019

ISSN: 2236-269X

DOI: 10.14807/ijmp.v10i5.897

The authors are thankful to Department of Mathematics; The University of Kalyani for providing financial assistance through UGC(SAP) Programme. This support is great fully acknowledged. We are also thankful to the reviewers for their helpful suggestions.

JEL Classification: C44, Y80, C61.

Mathematics Subject Classification: 90B05.

Conflict of Interest: We declare that there is no conflict of interest.

\section{REFERENCES :}

BELLMANN, R. E.; ZADEH, L. A. (1970) Decision Making in a Fuzzy Environment, Management Science, v. 17, n. 4 December.

BHUNIA, A. K.; SHAIKH, A. A. (2014) A deterministic inventory model for deteriorating items with selling price dependent demand and three-parameter Weibull distributed deterioration. International Journal of Industrial Engineering Computations, n. 5, p. 497-510.

DUBOIS, D.; PRADE, H. (1978) Operation on fuzzy number, International Journal of Systems Science, v. 9, n. 6, p. 613-626.

DUTTA, D.; KUMAR, P. (2012). Fuzzy Inventory Model without Shortage Using Trapezoidal Fuzzy Number with Sensitivity Analysis. IOSR Journal of Mathematics (IOSR-JM) ISSN: 2278-5728, v. 4, n. 3 (Nov. - Dec. ), p. 32-37.

GHARE, P. M.; SCHRADER, G. H. (1963) A model for exponentially decaying inventory system, International Journal of Production Research, v. 21, p. 449460.

GHOREISHI, M.; WEBER, G. W.; MIRZAZADEH, A. (2014) An inventory model for non-instantaneous deteriorating items with partial backlogging, permissible delay in payments, inflation- and selling price-dependent demand and customer returns,

Annals of Operations Research. DOI 10.1007/s10479-014-1739-7, 14 October.

ISLAM. S.; BISWAS, A. K. (2017) A Fuzzy Inventory Model having Exponential Demand with Weibull Distribution for Non-Instantaneous Deterioration, Shortages under Partially Backlogging and Time Dependent Holding Cost, International Journals of Advanced Research in Computer Science and Software Engineering, v. 7, n. 6, June.

MARAGATHAM, M.; LAKSMIDEVI P. K. (2014) A Fuzzy Inventory Model for Deteriorating Items with Price Dependent Demand, Intern. J. Fuzzy Mathematical Archive, v. 5, n. 1, 2014, p. 39-47, ISSN: 2320-3242 (P), 2320-3250 (online).

MISRA, R. B. (1975) Optimum Production lot-size model for a system with deteriorating inventory, International Journal of Production Research, n. 13, p. 495-505.

SYED, J. K.; AZIZ, L. A. (2007) Fuzzy Inventory Model without Shortages Using Signed Distance Method Applied, Mathematics \& Information Sciences, v. 1, n. 2, p. 203-209. 
ZADEH, L. A. (1965) Fuzzy sets, Information and Control, v. 8, n. 3, p. 338-353.

ZIMMERMAN, H. J. (1985) Application of fuzzy set theory to mathematical programming, Information Science, n. 36, p. 29-58. 